\title{
On muting mobile terminals for uplink interference mitigation in HetNets—system-level analysis via stochastic geometry
}

\author{
Francisco Javier Martín ${ }^{3}$, Xiaojun Xi², Marco Di Renzo ${ }^{2 *}$ (D), Mari Carmen Aguayo-Torres ${ }^{1}$ \\ and Gerardo Gomez ${ }^{1}$
}

\begin{abstract}
We investigate the performance of a scheduling algorithm where the mobile terminals (MTs) may be turned off if they cause a level of interference greater than a given threshold. This approach, which is referred to as interference aware muting (IAM), may be regarded as an interference-aware scheme that is aimed to reduce the level of interference. We analyze its performance with the aid of stochastic geometry and compare it against other interference-unaware and interference-aware schemes, where the level of interference is kept under control in the power control scheme itself rather than in the scheduling process. IAM is studied in terms of average transmit power, mean and variance of the interference, coverage probability, spectral efficiency (SE), and binary rate (BR), which accounts for the amount of resources allocated to the typical MT. Simplified expressions of SE and BR for adaptive modulation and coding schemes are proposed, which better characterize practical communication systems. Our system-level analysis unveils that IAM increases the BR and reduces the mean and variance of the interference. It is proved that an operating regime exists, where the performance of IAM is independent of the cell association criterion, which simplifies the joint design of uplink and downlink transmissions.
\end{abstract}

Keywords: Cellular networks, Scheduling, Stochastic geometry

\section{Methods/experimental}

The methods used in the paper are based on the mathematical tools of point processes and stochastic geometry. A new analytical framework for performance analysis is introduced. The theoretical frameworks are validated against Monte Carlo simulations.

\section{Introduction}

Interference awareness can be exploited at both the physical and medium access control (MAC) layers to boost the performance of mobile networks. It is especially useful in the uplink (UL) of heterogeneous cellular networks (HCNs) for interference mitigation and performance enhancement.

\footnotetext{
*Correspondence: marco.direnzo@|2s.centralesupelec.fr

${ }^{2}$ Laboratoire des Signaux et Systèmes, CNRS, CentraleSupelec, Univ Paris-Sud, Université Paris-Saclay, Paris, Gif-sur-Yvette, 91192 Paris, France

Full list of author information is available at the end of the article
}

In current HCNs, the mobile terminals (MTs) are associated with the same base station (BS) in the UL and downlink (DL) [1]. The cell association is performed based on DL pilot signals and the serving BS is chosen based on a given criterion, e.g., the highest average received power in the DL. In the UL, the same BS is used [1] which leads to a situation where MTs are associated with distant BSs. In this context, the use of fractional power control (FPC) accentuates the detrimental effect of the MTs that cause strong interference to neighboring BSs.

\subsection{UL analysis: state-of-the-art}

The aforementioned complex interactions between power control and association in the UL require accurate mathematical frameworks to gain insights about the performance trends and limitations of existing and future networks. Unfortunately, the mathematical analysis of the UL of HCNs is more involved than the analysis of the DL for 
two main reasons: (i) due to the use of power control, the transmit power of the MTs depends on the distance to their serving BSs and (ii) even though the locations of BSs and MTs are drawn from two independent Poisson point processes (PPPs), the locations of the interfering MTs scheduled in a given orthogonal resource block (RB) do not follow a PPP. These two peculiarities, as compared to the DL, make the mathematical analysis of the UL intractable without resorting to approximations [2]. In [3], the case of homogeneous cellular networks with FPC is studied. To avoid such a mathematical intractability, it is assumed that the MTs that are scheduled on a given RB form a Voronoi tessellation, and a single BS is available in each Voronoi cell. However, such an approach does not consider HCNs.

The case of the UL of HCNs is accurately modeled in recent works like [2, 4-6], where the spatial correlation between the location of the probe BS and those of the interfering MTs is considered.

In [4], a framework to model HCNs with a truncated channel inversion power control under the smallest path loss association is introduced. In this work, a homogeneous PPP is used as a generative process for the locations of the interfering MTs, but the spatial correlation is accounted for by means of an indicator function that discards interfering MTs' locations based on their received powers.

The case of UL and DL with decoupled access is considered in [2]. The association is based on maximum weighted received powers and FPC is considered in the UL. To account for the spatial correlations, a nonhomogeneous PPP is considered to model the locations of the interfering MTs.

A framework for the UL of HCNs with multi-antenna $\mathrm{BSs}$ is studied in [5]. In this work, FPC is considered under a generalized association criteria and two extreme detection techniques in terms of complexity and performance: maximum-ratio combining (MRC) and optimum combining (OC). It is demonstrated that $\mathrm{OC}$, which can be regarded as an interference-aware detection technique for multi-antenna receivers, greatly outperforms MRC when MTs use aggressive power control, i.e., when the interference is high. The spatial correlation is imposed by means of a conditional thinning that takes into account the generalized cell association procedure.

Interference-awareness is also studied in [6], which considers HCNs with single-antenna BSs. In this work, a power control mechanism [7] is studied, which is referred to as interference aware fractional power control (IAFPC). This approach consists of introducing a maximum interference level, $i_{0}$, that the transmission of each MT is allowed to cause to its most interfered BS.
In simple terms, the MTs adjust their transmit power in order to cause a maximum interference level of $i_{0}$ to their most interfered BS.

In the present paper, we investigate another option for interference mitigation in the UL and compare it with previously reported schemes. The approach consists of exploiting interference-awareness when scheduling the transmission of the MTs, rather than in the power control scheme itself (IAFPC) or in the detection process of the receiver $(\mathrm{OC})$. As a result, interference management is conducted at the MAC layer rather than at the physical layer. The considered approach is referred to as interference aware muting (IAM) and consists of turning off, i.e., muting, the MTs whose interference towards the most interfered BS is above a given threshold.

The main difference between IAFPC and IAM can be summarized as follows. In IAFPC, all the MTs are active and adjust their transmit power for interference mitigation. In IAM, on the other hand, the transmit power of the MTs does not account for any interference constraints but some MTs may not be allowed to transmit if they produce too much interference. As a result, IAM has the potential of reducing the aggregate interference in the UL and of enabling the active MTs to better use the available resources, i.e., the transmission bandwidth.

On the other hand, it reduces the fairness of allocating the resources among the MTs, since some of them may be turned off. Nevertheless, thanks to mobility and shadowing, the muted MTs are only inactive for a given period of time. Hence, from the perspective of MTs, the question to answer is whether this muting increases its achievable binary rate (BR), taking into account both the active and inactive periods. In the present paper, this issue is analyzed as well, and some conclusions are drawn.

\subsection{Technical contribution}

The main objective of the present paper is to quantify the advantages and the limitations of IAM and compare it against the IAFPC scheme. This issue is, however, mathematically challenging. In this paper, we overcome this mathematical intractability by using an approach similar to [5] and [6], which is referred to as conditional thinning. In simple terms, the locations of the active MTs are assumed to be drawn from a PPP but spatial constraints (correlations) are introduced, which account for the location of the serving BS, for the location of the most interfered BS, and for the maximum level of interference allowed. Based on these modeling assumptions, which are validated against extensive Monte Carlo simulations, we provide the following contributions.

- We study the IAM scheme in terms of average transmit power of the MTs, mean, and variance of the interference. The mathematical analysis reveals 
that IAM is capable of reducing the three latter performance metrics compared with IAFPC, which results in several advantages for practical implementations.

Reducing the variance of the interference, e.g., is beneficial for better estimating the SINR and, thus, for reducing the error probability of practical decoding schemes, e.g., turbo decoding, [8], and for making easier the selection of the most appropriate modulation and coding scheme (MCS) to use in LTE systems [7].

- To make our study and conclusions directly applicable to current communication systems that are based on adaptive modulation and coding (AMC) transmission, we provide tractable expressions of SE and BR based on practical MCSs that are compliant with the LTE standard and whose parameters are obtained from a link-level simulator $[9,10]$.

- With the aid of the proposed mathematical frameworks, we compare IAFPC and IAM schemes in terms of $\mathrm{SE}$ and $\mathrm{BR}$, which provide information on their strengths and weaknesses. The SE provides information on how well the MTs exploit the available resources (e.g., bandwidth) that are shared among the MTs served by the same BS, whereas the $\mathrm{BR}$ accounts for the specific fraction of resources that is allocated to each MT served by a given BS. While the IAFPC scheme is superior in terms of SE, the IAM scheme is superior in terms of RB. This implies that IAM provides service to fewer users, which get better performance compared with IAFPC. To characterize this trade-off, we investigate the fairness of both schemes, which is defined as the probability that a randomly chosen MT gets access to the resources, and provide a tractable frameworks for its analysis.

- In light of the emerging UL-DL decoupling principle, we develop the mathematical frameworks for a general cell association (GCA) criterion, whose association weights may be appropriately optimized for performance enhancement. By direct inspection of the mathematical frameworks, we prove that three operating regimes can be identified as a function of the interference threshold $i_{0}$ : (i) the first, where the performance is independent of $i_{0}$, (ii) the second, where the performance depends on $i_{0}$ but it does not depend on the cell association, and iii) the third, where the performance depends on $i_{0}$ and the cell association. Of particular interest in this paper is the second regime, which highlights that UL-DL decoupling may not be an issue for some system setups, which in turn simplifies the design of HCNs.

- As for the relevant case study for the UL where the serving BS of the typical MT is identified based on the smallest path loss association (SPLA) criterion with channel inversion power control [1], we provide simple and closed-form frameworks for relevant performance indicators and prove that two operating regimes exist: (i) the first, where the performance depends on $i_{0}$ (interference-aware) and (ii) the second, where the performance is independent of $i_{0}$ (interference-unaware). We prove, in addition, that (i) the scaling law of the average transmit power of the MTs, the mean interference, and the probability that a MT gets access to the resources is a polynomial function of $i_{0}$ whose exponent depends on the path loss exponent, (ii) the distance towards the serving BS gets smaller as $i_{0}$ increases, and (iii) the CCDF of the SINR is independent of the density of BSs.

To the best of authors' knowledge, all these contributions are new in the literature and are not included in previous works. For instance, the muting mechanism introduces further correlations that do not exist in [6] and need to be taken into account. This muting makes the whole analysis different. New metrics like the BR, which accounts for the amount of resources allocated by the scheduler, are obtained and a new framework to compute the $\mathrm{SE}$ and BR with AMC, which is closer to real systems than Shannon formula, is introduced as well. Finally, closed-form expressions and remarks are obtained which provide important insights about system performance, fairness, and cell association.

The remainder of this paper is organized as follows. Section 3 introduces the system model and the approach for system-level analysis. In Sections 4 and 5, the analysis of IAM is presented for GCA and SPLA criteria, respectively. The BR of AMC schemes is analyzed and discussed in Section 6. In Section 7, we introduce and study a hybrid scheme that allows us to overcome some fairness issues introduced by the IAM scheme. In Section 8, IAM and IAFPC schemes are compared against each other via numerical simulations and the main findings and performance trends derived in the paper are substantiated with the aid of Monte Carlo simulations. Finally, Section 9 concludes this paper.

Notation: A summary of the main symbols and functions used throughout the present paper is provided in Table 1 for the convenience of the readers.

\section{System model}

We consider the UL of a HCN composed of two tiers, $j \in$ $\mathcal{K}=\{1,2\}$, e.g., macro-and small-cell BSs, which are spatially distributed according to two independent PPPs, $\Phi^{(j)}$, of intensities $\lambda^{(j)}$. Each transmitted signal goes through an independent multi-path fading channel with Rayleigh fading and log-normal shadowing. The path loss is modeled by using a path loss slope $\tau$ and a path loss exponent 
Table 1 Summary of main symbols and functions used throughout the paper

\begin{tabular}{|c|c|}
\hline Symbol/function & Definition \\
\hline${ }_{2} F_{1}(\cdot, \cdot, \cdot, \cdot)$ & Gauss hypergeometric function \\
\hline $\mathcal{K}=\{1,2\}$ & $\begin{array}{l}\text { Tier set: tier } 1 \text { is related to macro BSs, and tier } 2 \text { is } \\
\text { related to small cell BSs }\end{array}$ \\
\hline$\tilde{j}=\{k \in \mathcal{K}: k \neq j\}$ & Complementary tier, i.e., $\tilde{1}=2$ and $\tilde{2}=1$ \\
\hline$\Phi^{(j)}, \lambda^{(j)}$ & $\begin{array}{l}\text { PPP and its density related to the locations of } \\
\text { macro }(j=1) \text { and small cell BSs }(j=2)\end{array}$ \\
\hline$\lambda_{\mathrm{MT}}$ & Density of the PPP of MTs' positions \\
\hline$\Phi, \lambda$ & PPP and its density related to the locations of all BSs \\
\hline$t^{(j)}$ & Association weight for tier $j$ \\
\hline$i_{0}, p_{0}, \epsilon, p_{\max }$ & $\begin{array}{l}\text { Interference threshold, target receive power, partial } \\
\text { compensation factor, and maximum transmit power }\end{array}$ \\
\hline$\tau, \alpha$ & Path loss slope and path loss exponent \\
\hline $\mathrm{MT}_{0}, \mathrm{MT}_{\mathrm{i}}$ & $\begin{array}{l}\text { Position of the probe MT and position of a generic } \\
\text { MT, e.g., an interfering MT }\end{array}$ \\
\hline$\Psi^{(k)}$ & PPP of interfering MTs's locations \\
\hline$R_{x,(q)}^{(j)}$ & $\begin{array}{l}\text { Distance (including shadowing) between } \\
\text { location } x \text { and the qth nearest BS from tier } j\end{array}$ \\
\hline$R_{\mathrm{MT}_{\mathrm{i}}}, U_{\mathrm{MT}_{\mathrm{i}}}, D_{\mathrm{MT}_{\mathrm{i}}}$ & $\begin{array}{l}\text { Distances (including shadowing) between MTi and } \\
\text { its serving BS, its most interfered BS and the probe BS }\end{array}$ \\
\hline$H_{M_{i}}$ & $\begin{array}{l}\text { Power gain of the multi-path fading which is } \\
\text { exponentially distributed }\end{array}$ \\
\hline$p_{\mathrm{MT}}(r)=p_{0}(\tau r)^{\alpha \epsilon}$ & $\begin{array}{l}\text { Transmit power for a given distance towards the } \\
\text { serving BS for active MTs. Muted MTs has } 0 \\
\text { transmit power }\end{array}$ \\
\hline$\sigma_{n}^{2}, 1$ & $\begin{array}{l}\text { Noise power and aggregate interference } \\
\text { according to Assumption } 1\end{array}$ \\
\hline $\mathcal{X}_{\mathrm{MT}_{i}}^{(j)}$ & Event defined as $M T_{i}$ is associated with tier $j$ \\
\hline $\mathcal{Q}_{\mathrm{MT}_{i}}^{(m)}$ & $\begin{array}{l}\text { Event defined as the most interfered } \mathrm{BS} \text { of } \mathrm{M} T_{i} \\
\text { belongs to tier } m\end{array}$ \\
\hline $\mathcal{X}_{\mathrm{MT}_{i}}^{(j, m)}$ & $\begin{array}{l}\text { Event defined as } \mathrm{MT} T_{j} \text { is associated with tier } j \\
\text { and the most interfered } \mathrm{BS} \text { of } \mathrm{M} T_{i} \text { belongs to tier } m\end{array}$ \\
\hline $\mathcal{A}_{\mathrm{MT}_{i}}$ & Event defined as $M T_{i}$ is active, i.e., non-muted \\
\hline$\overline{\mathcal{A}_{\mathrm{MT}_{i}}}$ & Event defined as $\mathrm{M} T_{i}$ is muted \\
\hline \multirow[t]{2}{*}{$\mathcal{O}_{\mathrm{MT}_{i}}^{(j, k)}$} & $\begin{array}{l}\text { Event defined as the interfering } \mathrm{M} T_{i} \text { of tier } \\
k \text { receives higher weighted average power }\end{array}$ \\
\hline & $\begin{array}{l}\text { from its serving BS than from the probe BS that } \\
\text { belong to tier } j\end{array}$ \\
\hline $\mathcal{Z}_{\mathrm{MT}_{i}}$ & $\begin{array}{l}\text { Event defined as the interfering } \mathrm{M} T_{i} \text { causes a } \\
\text { level of interference less than } i_{0} \text { to the probe } \mathrm{BS}\end{array}$ \\
\hline$f_{X}(\cdot)$ & $\begin{array}{l}\text { PDF (Probability Density Function) of random } \\
\text { variable } X\end{array}$ \\
\hline $\bar{F}_{X}(\cdot)$ & $\begin{array}{l}\text { CCDF (Complementary Cumulative Distribution } \\
\text { Function) of random variable } X\end{array}$ \\
\hline $\mathcal{L}_{X}(\cdot)$ & Laplace transform of random variable $X$ \\
\hline$f^{\prime}\left(x_{0}\right), f^{\prime \prime}\left(x_{0}\right)$ & $\begin{array}{l}\text { First and second derivatives of function } f(x) \\
\text { evaluated at } x=x_{0}\end{array}$ \\
\hline $\mathbb{E}[\cdot], \operatorname{Pr}(\cdot), \mathbf{1}(\cdot)$ & $\begin{array}{l}\text { Expectation operator, probability measure and } \\
\text { indicator function }\end{array}$ \\
\hline$\Gamma(z), \Gamma(a, z)$ & $\begin{array}{l}\text { Euler gamma function and incomplete gamma } \\
\text { function }\end{array}$ \\
\hline
\end{tabular}

$\alpha>2^{1}$. The cell association among MTs and BSs is based on the weighted average received power criterion, similar to [2], where the association weights are denoted by $t^{(j)}$ for tier $j \in \mathcal{K}$. Hence, the $i$ th MT is associated with the $n$th BS of tier $j$ if the MT is in the weighted Voronoi cell of $\mathrm{BS}_{n}^{(j)}$ with respect to $\Phi=\bigcup_{j \in \mathcal{K}} \Phi^{(j)}$. With these assumptions, shadowing can be modeled as a random displacement [11] of $\Phi^{(j)}[6,12]$.

For ease of writing, we introduce the event $\mathcal{X}_{\mathrm{MT}_{i}}^{(j)}$ as follows:

Definition 1 The event $\mathcal{X}_{M T_{i}}^{(j)}$ is defined as " $M T_{i}$ is associated with tier j."

In mathematical terms, therefore, the association criterion can be formulated as follows:

$$
\mathcal{X}_{\mathrm{MT}_{i}}^{(j)}=\left\{t^{(j)}\left(\tau R_{\mathrm{MT}_{i},(1)}^{(j)}\right)^{-\alpha}>t^{(\tilde{j})}\left(\tau R_{\mathrm{MT}_{i},(1)}^{(\tilde{j})}\right)^{-\alpha}\right\}
$$

where $\left(\tau R_{\mathrm{MT}}\right)^{-\alpha}$ is the path loss at a distance ${ }^{2} R_{\mathrm{MT}}$ from the transmitter, $\tilde{j}=\{k \in \mathcal{K}: k \neq j\}$ is the complementary tier of $j$, i.e., $\tilde{1}=2$, and $\tilde{2}=1, R_{x,(q)}^{(\tilde{j})}$ is the distance from $x$ to the $q$ th nearest BS of tier $\tilde{j}$, i.e., $R_{x,(1)}^{(\tilde{j})}$ is the distance to the nearest BS. The association weights $t^{(1)}$ and $t^{(2)}$ allow us to model the GCA criterion, which encompasses the SPLA criterion for $t^{(1)}=t^{(2)}$.

Throughout this paper, the analysis is performed for the probe or typical MT, i.e., for a randomly chosen MT, which is denoted by $\mathrm{MT}_{0}$. Its serving $\mathrm{BS}$ is referred to as the probe BS.

\subsection{Scheduling}

We consider full-frequency reuse, where all the BSs share the same bandwidth. Each BS has available a bandwidth of $b_{w}$ Hertz that is shared among the MTs that are in its Voronoi cell. In practice, $b_{w}$ is divided in orthogonal RBs and each scheduled MT in each cell transmits in one (or several) of these RBs. Thus, no intra-cell interference is available. This implies that a single MT per BS can interfere with the probe MT. The set of active interfering MTs of tier $k$ that are scheduled for transmission in a given $\mathrm{RB}$ is denoted by $\Psi^{(k)}$. For tractability, we assume that the number of RBs is large enough to be regarded as a continuous resource by the scheduler.

Based on these assumptions, the scheduling process of every BS consists of two steps:

1 To determine the set of active MTs. The active transmitters are the MTs that, simultaneously, cause less interference than $i_{0}$ to any BSs and that transmit with less power than $p_{\max }$. The MTs that do not fulfill these two constraints are turned off (muted). 
2 Resource allocation. Once the active MTs in each cell are identified, the bandwidth of each BS is equally divided among the active MTs associated with it. Let $N_{\mathrm{BS}_{n}^{(j)}}^{\mathcal{A}}$ be the number of MTs associated with $\mathrm{BS} \mathrm{BS}_{n}^{(j)}$. Each of them is allocated a bandwidth $b_{w} / N_{\mathrm{BS}_{n}^{(j)}}^{\mathcal{A}}{ }^{3} \mathrm{~Hz}$

This scheduling process characterizes the IAM scheme and makes it different from the IAFPC scheme in [6]. In [6], all the MTs are active and power control is responsible for controlling the level of interference, by making sure that the interference level at any BS is less than $i_{0}$.

To better understand the implications of interference awareness on turning off (muting) some MTs, we analyze the case study $i_{0} \rightarrow \infty$ as well, which is referred to as interference-unaware muting (IUM) ${ }^{4}$.

For ease of writing, we introduce some definitions that are useful for mathematical analysis.

Definition 2 The event $\mathcal{Q}_{M T_{i}}^{(m)}$ is defined as "the most interfered BS of $M T_{i}$ belongs to tier $m$."

Definition 3 The event $\mathcal{X}_{M T_{i}}^{(j, m)}=\mathcal{X}_{M T_{i}}^{(j)} \cap \mathcal{Q}_{M T_{i}}^{(m)}$ is defined as " $M T_{i}$ is associated with tier $j$ and the most interfered BS of $M T_{i}$ belongs to tier $m$."

In mathematical terms, $\mathcal{X}_{\mathrm{MT}_{i}}^{(j, m)}$ can be formulated as follows:

$$
\begin{aligned}
& \mathcal{X}_{\mathrm{MT}_{i}}^{(j, m)}=\mathcal{X}_{\mathrm{MT}_{i}}^{(j)} \cap \overbrace{\left\{R_{\mathrm{MT}_{i},(2)}^{(j)}>R_{\mathrm{MT}_{i},(1)}^{(m)}\right\}}^{\mathcal{Q}_{\mathrm{MT}_{i}}^{(m)}}, \text { if } j \neq m \\
& \mathcal{X}_{\mathrm{MT}_{i}}^{(j, m)}=\mathcal{X}_{\mathrm{MT}_{i}}^{(j)} \cap \overbrace{\left\{R_{\mathrm{MT}_{i},(2)}^{(j)}<R_{\mathrm{MT}_{i},(1)}^{(\tilde{j})}\right\}}^{\mathcal{Q}_{\mathrm{MT}_{i}}^{(m)}}, \text { if } j=m
\end{aligned}
$$

According to IAM, the MTs that either cause higher interference than $i_{0}$ or transmit with higher power than $p_{\max }$ are kept silent. The set of active MTs is defined as follows:

Definition 4 The event $\mathcal{A}_{M T_{i}}$ is defined as " $M T_{i}$ is active"

In mathematical terms, $\mathcal{A}_{\mathrm{MT}_{i}}$ can be formulated as follows:

$$
\begin{aligned}
\mathcal{A}_{\mathrm{MT}_{i}} & =\left\{\left(p_{\mathrm{MT} T}\left(R_{\mathrm{MT}_{i}}\right)<i_{0}\left(\tau U_{\mathrm{MT}_{i}}\right)^{\alpha}\right.\right. \\
& \left.\left.\cap p_{\mathrm{M} T}\left(R_{\mathrm{MT}_{i}}\right)<p_{\max }\right)\right\}
\end{aligned}
$$

where $p_{\mathrm{M} T}(r), p_{0}$, and $\epsilon$ are related to power control, and they are described in Table $1, R_{\mathrm{MT}_{i}}$ is the distance between $\mathrm{MT}_{\mathrm{i}}$ and its serving $\mathrm{BS}$, and $U_{\mathrm{MT}_{i}}$ is the distance between $\mathrm{MT}_{\mathrm{i}}$ and its most interfered BS. If the probe MT is associated with tier $j$, i.e., the event $\mathcal{X}_{\mathrm{MT}_{i}}^{(j)}$ is true, then $R_{\mathrm{MT}_{i}}=$
$R_{\mathrm{MT}_{i},(1)}^{(j)}$. The distance $U_{\mathrm{MT}_{i}}$ depends, on the other hand, on the event $\mathcal{X}_{\mathrm{MT}_{i}}^{(j, m)}$. Accordingly, $U_{\mathrm{MT}_{i}}=R_{\mathrm{MT}_{i},(1)}^{(m)}$ if $j \neq m$ and $U_{\mathrm{MT}_{i}}=R_{\mathrm{MT}_{i}(2)}^{(j)}$ if $j=m$. The aim of event $\mathcal{A}_{\mathrm{M} T_{i}}$ is to capture the spatial correlation between the position of a given MT, its serving BS, and its most interfered BS, which follows from the muting process.

As far as IAM is concerned, fractional power control is applied at the physical layer and is interference-unaware, i.e., the transmit power of the MTs that are not turned off depends only on path loss and shadowing and it can be expressed as $p_{\mathrm{MT}}\left(R_{\mathrm{MT}_{0}}\right)$. If the MTs are muted, on the other hand, their transmit power is equal to zero. This implies that their associated $\operatorname{SINR}, \mathrm{BR}$, etc. are, by definition, equal to zero as well.

\subsection{SINR}

The SINR of the typical active MT that is measured at the probe BS can be formulated as

$$
\operatorname{SINR}_{\mathrm{MT}_{0}}=\frac{H_{\mathrm{MT}_{0}}\left(\tau R_{\mathrm{MT}_{0}}\right)^{-\alpha} p_{\mathrm{MT}}\left(R_{\mathrm{MT}_{0}}\right)}{I+\sigma_{n}^{2}}
$$

where $H_{\mathrm{MT}_{0}}$ is the channel gain, $R_{\mathrm{MT}_{0}}$ is the distance from the serving BS, $p_{\mathrm{MT}}\left(R_{\mathrm{MT}_{0}}\right)$ is the transmit power, $I$ is the other-cell interference, and $\sigma_{n}^{2}$ is the noise power.

In the UL, as discussed in Section 2, the set of interfering MTs does not constitute a PPP, even though the MTs and BSs are distributed according to a PPP. Further details can be found in [5] and [6]. This makes the mathematical analysis intractable. In the present paper, the distinctive scheduling process of IAM negatively affects the mathematical tractability of the problem at hand even further. To make the analysis tractable, some approximations for modeling the set of active MTs are needed. In [5] and [6], it is shown that a tractable approximation consists of assuming that the set of active MTs can still be modeled as a PPP, provided that appropriate spatial constraints on the locations of the MTs are introduced. Stated differently, the set of active MTs is modeled as a spatially-thinned PPP or equivalently as a non-homogeneous PPP.

Before introducing the approach to model interfering MTs' locations, the following events need to be defined:

Definition 5 The event $\mathcal{O}_{M T_{i}}^{(j, k)}$ is defined as "the interfering $M T_{i}$ of tier $k$ receives higher weighted average power from its serving BS than from the probe BS that belongs to tier j."

In mathematical terms, $\mathcal{O}_{\mathrm{MT}_{i}}^{(j, k)}$ can be formulated as follows:

$$
\mathcal{O}_{\mathrm{MT}_{i}}^{(j, k)}=\left\{t^{(k)}\left(\tau R_{\mathrm{MT}_{i}}\right)^{-\alpha}>t^{(j)}\left(\tau D_{\mathrm{MT}_{i}}\right)^{-\alpha}\right\}
$$


Definition 6 The event $\mathcal{Z}_{M T_{i}}$ is defined as "the interfering $M T_{i}$ causes a level of interference less than $i_{0}$ to the probe BS."

In mathematical terms, $\mathcal{Z}_{\mathrm{MT}_{i}}$ can be formulated as follows:

$$
\mathcal{Z}_{\mathrm{MT}_{i}}=\left\{p_{\mathrm{MT}}\left(R_{\mathrm{MT}_{i}}\right)\left(\tau D_{\mathrm{MT}_{i}}\right)^{-\alpha}<i_{0}\right\}
$$

Hence, inspired by [5] and [6], our mathematical framework is based on the following approximation.

Assumption 1 The other-cell interference of the typical active MT is approximated as [6]

$$
I \approx \sum_{k \in \mathcal{K}} \sum_{M T_{i} \in \Psi^{(k)}} \frac{H_{M T_{i}} p_{M T}\left(R_{M T_{i}}\right)}{\left(\tau D_{M T_{i}}\right)^{\alpha}} \mathbf{1}\left(\mathcal{O}_{M T_{i}}^{(j, k)}\right) \mathbf{1}\left(\mathcal{Z}_{M T_{i}}\right)
$$

where $\Psi^{(k)}$ is a PPP of intensity $\lambda^{(k)}$ whose points constitute the locations of the interfering MTs that are scheduled for transmission in the same $R B$ as that of the typical MT, the events $\mathcal{O}_{M T_{i}}^{(j, k)}$ and $\mathcal{Z}_{M T_{i}}$ take into account the necessary spatial constraints imposed by the cell association criterion and the maximum interference and power constraints, respectively, $R_{M T_{i}}$ and $D_{M T_{i}}$ are the distances from $M T_{i}$ to its own serving $B S$ and to the probe BS, respectively.

More specifically, (i) the event $\mathcal{O}_{\mathrm{MT}_{i}}^{(j, k)}$ is necessary to account for the spatial correlation that exists between the locations of the probe BS, the interfering MTs and their serving BSs, since the interfering MTs must lie outside the Voronoi cell of the probe BS by definition of cell association, and (ii) the event $\mathcal{Z}_{\mathrm{MT}_{i}}$ is necessary to account for the fact that the interfering MTs need to cause less interference than $i_{0}$ according to the IAM scheduling process.

The next two sections provide mathematical expressions of the CCDF of the SINR and of the mean and variance of the other-cell interference for GCA and SPLA cell association criteria respectively.

\section{General cell association criterion}

We start introducing some enabling results for proving the main theorems of this section.

Proposition 1 The probability that the typical MT is active and is associated with tier $j$ is

$$
\begin{aligned}
\operatorname{Pr}\left(\mathcal{A}_{M T_{0}}\right) & =\sum_{j \in \mathcal{K}} \int_{v>0} \mathbf{1}\left(v<\frac{1}{\tau}\left(\frac{p_{\max }}{p_{0}}\right)^{\frac{1}{\alpha}}\right) \\
& \times\left(v^{(j)}(v)+\eta^{(j)}(v)\right) \mathrm{d} v
\end{aligned}
$$

where $v^{(j)}(v)$ and $\eta^{(j)}(v)$ are defined in (9) and (10), respectively.

$$
\begin{aligned}
& v^{(j)}(v)=2 \pi v \lambda^{(j)}\left(\mathrm{e}^{-\pi \lambda^{(j)} v^{2}} \times\right. \\
& \left(\mathrm{e}^{-\pi \lambda^{\tilde{j})} \max \left(\left(\frac{p_{0}}{i_{0}}\right)^{\frac{1}{\alpha}} \frac{(\tau \nu)^{\epsilon}}{\tau},\left(\frac{t^{(\tilde{j})}}{t^{(j)}}\right)^{\frac{1}{\alpha}} v\right)}-\mathrm{e}^{-\pi \lambda^{(\tilde{j})} v^{2}}\right) \times \\
& \mathbf{1}\left(v>\max \left(\left(\frac{p_{0}}{i_{0}}\right)^{\frac{1}{\alpha}} \frac{(\tau v)^{\epsilon}}{\tau},\left(\frac{t^{(\tilde{j})}}{t^{(j)}}\right)^{\frac{1}{\alpha}} v\right)\right) \\
& \left.+\frac{\lambda^{(\tilde{j})}}{\lambda^{(j)}+\lambda(\tilde{j})} \mathrm{e}^{-\pi\left(\lambda^{(j)}+\lambda^{\tilde{j})}\right) \max ^{2}\left(\left(\frac{p_{0}}{i_{0}}\right)^{\frac{1}{\alpha}} \frac{(\tau v)^{\epsilon}}{\tau},\left(\frac{t^{\tilde{j})}}{t^{(j)}}\right)^{\frac{1}{\alpha}} v, v\right)}\right)
\end{aligned}
$$

$$
\begin{aligned}
& \eta^{(j)}(v)=2 \pi v \lambda^{(j)}\left(\mathrm{e}^{-\pi \lambda^{(\tilde{j})}\left(\frac{t^{(\tilde{j})}}{t^{(j)}}\right)^{\frac{2}{\alpha}} v^{2}} \times\right. \\
& \left(\mathrm{e}^{-\pi \lambda^{(j)} \max ^{2}\left(\left(\frac{p_{0}}{i_{0}}\right)^{\frac{1}{\alpha} \frac{(\tau \nu)^{\epsilon}}{\tau}, v}\right)}-\mathrm{e}^{-\pi \lambda^{(j)}\left(\frac{t^{(\tilde{j})}}{t^{(j)}}\right)^{\frac{2}{\alpha}} v^{2}}\right) \times \\
& \mathbf{1}\left(v>\left(\frac{t^{(j)}}{t^{(\tilde{j})}}\right)^{\frac{1}{\alpha}} \max \left(\left(\frac{p_{0}}{i_{0}}\right)^{\frac{1}{\alpha}} \frac{(\tau v)^{\epsilon}}{\tau}, v\right)\right) \\
& \left.+\frac{\lambda^{(j)}}{\lambda^{(j)}+\lambda^{(\tilde{j})}} \mathrm{e}^{-\pi\left(\lambda^{(j)}+\lambda^{(\tilde{j})}\right) \max ^{2}\left(\left(\frac{p_{0}}{i_{0}}\right)^{\frac{1}{\alpha}} \frac{(\tau v)^{\epsilon}}{\tau}, v,\left(\frac{t^{(j)}}{t^{(j)}}\right)^{\frac{1}{\alpha}} v\right)}\right)
\end{aligned}
$$

Proposition 1 is useful for understanding and quantifying the fairness of the IAM scheme. The higher $\operatorname{Pr}\left(\mathcal{A}_{\mathrm{MT}_{0}}\right)$ is, in fact, the higher the probability that a randomly chosen MT is served in a given RB and, thus, the higher the fairness that it gets access to the available resources is ${ }^{5}$.

\section{Proof See Appendix A.}

Lemma 1 The probability density function (PDF) of the distance between the typical MT and its serving BS by conditioning on the event $\mathcal{X}_{M T_{0}}^{(j, m)} \cap \mathcal{A}_{M T_{0}}$ can be formulated as follows: 


$$
\begin{aligned}
& f_{R_{M T_{0}}}\left(v \mid \mathcal{X}_{M T_{0}}^{(j, m)}, \mathcal{A}_{M T_{0}}\right)= \\
& \begin{cases}\frac{v^{(j)}(v)}{\operatorname{Pr}\left(\mathcal{X}_{M T_{0}}^{(j, m)}, \mathcal{A}_{M T_{0}}^{(j, m)}\right)}, v<\frac{1}{\tau}\left(\frac{p_{\text {max }}}{p_{0}}\right)^{\frac{1}{\alpha \epsilon}}, \quad \text { if } j \neq m \\
\frac{\eta^{(j)}(v)}{\operatorname{Pr}\left(\mathcal{X}_{M T_{0}}^{(j,)}, \mathcal{A}_{M T_{0}}^{(j, j)}\right)}, v<\frac{1}{\tau}\left(\frac{p_{\max }}{p_{0}}\right)^{\frac{1}{\alpha \epsilon}}, \quad \text { if } j=m\end{cases}
\end{aligned}
$$

where $v^{(j)}(v)$ and $\eta^{(j)}(v)$ are defined in (9) and (10), respectively.

Proof The Cumulative Distribution Function (CDF) of the distance between the typical MT and its serving BS by conditioning on the MT being active and on $\mathcal{X}_{\mathrm{MT}_{0}}^{(j, m)}$ is obtained by using steps similar to Appendix A. The PDF is obtained from the CDF by computing the derivative.

In the UL, an important performance metric to study is the average transmit power of the typical MT, which is related to its power consumption. Since some MTs may be turned off in the IAM scheme, this implies that some MTs may transmit zero power, which results in reducing their power consumption. The following proposition provides the average transmit power of the typical MT, by taking into account that the typical MT may be a MT that is turned off as it does not fulfill either the maximum power constraint or the maximum interference constraint.

Proposition 2 The average transmit power of the typical MT can be formulated as follows:

$$
\begin{aligned}
& \mathbb{E}\left[P_{M T_{0}}\right]=\sum_{j \in \mathcal{K}} \sum_{m \in \mathcal{K}} \operatorname{Pr}\left(\mathcal{X}_{M T_{0}}^{(j, m)}, \mathcal{A}_{M T_{0}}\right) \int_{p=0}^{\infty} \frac{p}{\tau p_{0} \alpha \epsilon} \\
& \times\left(\frac{p}{p_{0}}\right)^{\frac{1}{\alpha \epsilon}-1} f_{R_{M T_{0}}}\left(\frac{1}{\tau}\left(\frac{p}{p_{0}}\right)^{\frac{1}{\alpha \epsilon}} \mid \mathcal{X}_{M T_{0}}^{(j, m)}, \mathcal{A}_{M T_{0}}\right) \mathrm{d} p
\end{aligned}
$$

where $f_{R_{M T_{0}}}\left(v \mid \mathcal{X}_{M T_{0}}^{(j, m)}, \mathcal{A}_{M T_{0}}\right)$ is in (11) and $\operatorname{Pr}\left(\mathcal{X}_{M T_{0}}^{(j, m)}, \mathcal{A}_{M T_{0}}\right)$ is defined in Appendix A.

Proof It follows by computing the average transmit power by conditioning on the events $\mathcal{A}_{\mathrm{MT}_{0}}$ and $\mathcal{X}_{\mathrm{MT}_{0}}^{(j, m)}$. The final result is obtained from the total probability theorem.

Remark 1 (Exact analysis) The previous propositions and lemmas are exact, since they do not depend on the set of active interfering MTs but depend only on the locations of the BSs, which constitute a PPP, and on the typical MT. In other words, Assumption 1 is not applied.
The next lemma provides the Laplace transform of the other-cell interference based on its mathematical formulation in (7), which exploits Assumption 1.

Lemma 2 Assume that the typical MT is associated with a BS of tier $j$. The Laplace transform of the (conditional) interference in (7) can be formulated as follows:

$$
\mathcal{L}_{I}\left(s \mid \mathcal{X}_{M T_{0}}^{(j)}\right)=\exp \left(\beta^{(j)}(s)\right)
$$

where $\beta^{(j)}(s)$ is defined as follows:

$$
\begin{gathered}
\beta^{(j)}(s)=-\sum_{k \in \mathcal{K}} 2 \pi \lambda^{(k)} \sum_{n \in \mathcal{K}} \operatorname{Pr}\left(\mathcal{Q}_{M T_{i}}^{(n)} \mid \mathcal{X}_{M T_{i}}^{(k)}, \mathcal{A}_{M T_{i}}\right) \\
\int_{0}^{\infty} f_{R_{M T_{i}}}\left(r \mid \mathcal{X}_{M T_{i}}^{(k, n)}, \mathcal{A}_{M T_{i}}\right) \chi(s, r) \mathrm{d} r
\end{gathered}
$$

$f_{R_{M T_{i}}}\left(r \mid \mathcal{X}_{M T_{i}}^{(k, n)}, \mathcal{A}_{M T_{i}}\right)$ is the PDF of the distance between the ith interfering $M T$ and its serving $B S$, which is provided in Lemma 1, $\chi(s, r)$ is defined as follows:

$$
\begin{aligned}
& \chi(s, r)=\frac{p_{0} s(\tau r)^{\alpha \epsilon} \tau^{-\alpha}}{\alpha-2} \\
& \times \max ^{2-\alpha}\left(\left(\frac{t^{(j)}}{t^{(k)}}\right)^{\frac{1}{\alpha}} r,\left(\frac{p_{0}}{i_{0}}\right)^{\frac{1}{\alpha}} \frac{(\tau r)^{\epsilon}}{\tau}\right) \\
& \times_{2} \mathrm{~F}_{1}\left(1, \frac{\alpha-2}{\alpha}, 2-\frac{2}{\alpha},-p_{0} s(\tau r)^{\alpha \epsilon} \tau^{-\alpha}\right. \\
& \left.\max ^{-\alpha}\left(\left(\frac{t^{(j)}}{t^{(k)}}\right)^{\frac{1}{\alpha}} r,\left(\frac{p_{0}}{i_{0}}\right)^{\frac{1}{\alpha}} \frac{(\tau r)^{\epsilon}}{\tau}\right)\right)
\end{aligned}
$$

and $\operatorname{Pr}\left(\mathcal{Q}_{M T_{i}}^{(n)} \mid \mathcal{X}_{M T_{i}}^{(k)}, \mathcal{A}_{M T_{i}}\right)$ is defined as follows:

$$
\begin{aligned}
& \operatorname{Pr}\left(\mathcal{Q}_{M T_{i}}^{(n)} \mid \mathcal{X}_{M T_{i}}^{(k)}, \mathcal{A}_{M T_{i}}\right)=\frac{\operatorname{Pr}\left(\mathcal{X}_{M T_{i}}^{(k, n)}, \mathcal{A}_{M T_{i}}\right)}{\operatorname{Pr}\left(\mathcal{X}_{M T_{i}}^{(k)}, \mathcal{A}_{M T_{i}}\right)} \\
& =\frac{\operatorname{Pr}\left(\mathcal{X}_{M T_{i}}^{(k, n)}, \mathcal{A}_{M T_{i}}\right)}{\sum_{q \in \mathcal{K}} \operatorname{Pr}\left(\mathcal{X}_{M T_{i}}^{(k, q)}, \mathcal{A}_{M T_{i}}\right)}
\end{aligned}
$$

Proof See Appendix B.

From the Laplace transform in (47), the moments of the interference can be obtained as shown in the next proposition. Of particular interest is the variance of the interference, since its affects the performance of AMC schemes [7]: the smaller the variance is, the more robust and accurate the estimation of the SINR is, which makes easier the choice of the best MCS to use. 
Proposition 3 The mean and variance of the interference can be formulated as follows:

$$
\begin{aligned}
\mathbb{E}[I] & =-\sum_{j \in \mathcal{K}} \operatorname{Pr}\left(\mathcal{X}_{M T_{0}}^{(j)}\right) \beta^{\prime(j)}(0) \\
\operatorname{var}(I) & =-\sum_{j \in \mathcal{K}} \operatorname{Pr}\left(\mathcal{X}_{M T_{0}}^{(j)}\right) \\
& \left(\beta^{\prime \prime(j)}(0)+\left(\beta^{\prime(j)}(0)\right)^{2}-(\mathbb{E}[I])^{2}\right)
\end{aligned}
$$

where the following definitions hold:

$$
\begin{aligned}
\beta^{\prime(j)}(0) & =-\sum_{k \in \mathcal{K}} 2 \pi \lambda^{(k)} \sum_{n \in \mathcal{K}} \operatorname{Pr}\left(\mathcal{Q}_{M T_{i}}^{(n)} \mid \mathcal{X}_{M T_{i}}^{(k)}, \mathcal{A}_{M T_{i}}\right) \\
& \int_{r=0}^{\infty} f_{R_{M T_{i}}}\left(r \mid \mathcal{X}_{M T_{i}}^{(k, n)}, \mathcal{A}_{M T_{i}}\right) \frac{p_{0}(\tau r)^{\alpha \epsilon} \tau^{-\alpha}}{\alpha-2} \\
& \max ^{2-\alpha}\left(\left(\frac{t^{(j)}}{t^{(k)}}\right)^{\frac{1}{\alpha}} r,\left(\frac{p_{0}}{i_{0}}\right)^{\frac{1}{\alpha}} \frac{(\tau r)^{\epsilon}}{\tau}\right) \mathrm{d} r \\
\beta^{\prime \prime(j)}(0) & =-\sum_{k \in \mathcal{K}} 2 \pi \lambda^{(k)} \sum_{n \in \mathcal{K}} \operatorname{Pr}\left(\mathcal{Q}_{M T_{i}}^{(n)} \mid \mathcal{X}_{M T_{i}}^{(k)}, \mathcal{A}_{M T_{i}}\right) \\
& \times \int_{r=0}^{\infty} f_{R_{M T_{i}}}\left(r \mid \mathcal{X}_{M T_{i}}^{(k, n)}, \mathcal{A}_{M T_{i}}\right)^{\frac{p_{0}^{2}}{(\tau r)^{2 \alpha \epsilon} \tau^{-2 \alpha}}} \\
& \max ^{2(1-\alpha)}\left(\left(\frac{t^{(j)}}{t^{(k)}}\right)^{\frac{1}{\alpha}} r,\left(\frac{p_{0}}{i_{0}}\right)^{\frac{1}{\alpha}} \frac{(\tau r)^{\epsilon}}{\tau}\right) \mathrm{d} r
\end{aligned}
$$

Proof It directly follows from the first and second derivative of 47 evaluated at $s=0$.

Remark 2 (Impact of $i_{0}$ ) By inspection of Propositions 2 and 3, we evince that the average transmit power, the mean, and variance of the interference decrease by decreasing $i_{0}$. Since the interference-unaware setup is obtained by setting $i_{0} \rightarrow \infty$, this implies that IAM is beneficial in terms of reducing the power consumption of the MTs and of implementing AMC schemes. The system fairness may, however, be negatively affected if $i_{0}$ decreases, as more MTs are muted.

The next theorem provides a tractable expression of the coverage probability of $\mathrm{HCNs}$.
Theorem 1 The CCDF of the SINR of the typical MT can be formulated as follows:

$$
\begin{aligned}
& \bar{F}_{S I N R}(\gamma)=\sum_{j \in \mathcal{K}} \sum_{m \in \mathcal{K}} \operatorname{Pr}\left(\mathcal{X}_{M T_{0}}^{(j, m)}, \mathcal{A}_{M T_{0}}\right) \\
& \times \int_{\nu=0}^{\infty} f_{R_{M T_{0}}}\left(v \mid \mathcal{X}_{M T_{0}}^{(j, m)}, \mathcal{A}_{M T_{0}}\right) \\
& \mathrm{e}^{-\gamma \sigma_{n}^{2}(\tau v)^{\alpha(1-\epsilon)}} p_{0}^{-1} \mathcal{L}_{I}\left(\gamma(\tau \nu)^{\alpha(1-\epsilon)} p_{0}^{-1} \mid \mathcal{X}_{M T_{0}}^{(j)}\right) \mathrm{d} v
\end{aligned}
$$

Proof With the aid of the total probability theorem, we have:

$$
\begin{aligned}
\bar{F}_{\mathrm{SINR}} & (\gamma)=\bar{F}_{\mathrm{SINR}}\left(\gamma \mid \mathcal{A}_{\mathrm{MT}_{0}}\right) \operatorname{Pr}\left(\mathcal{A}_{\mathrm{MT}_{0}}\right)+0 \times \operatorname{Pr}\left(\overline{\mathcal{A}_{\mathrm{MT}_{0}}}\right) \\
= & \sum_{j \in \mathcal{K}} \sum_{m \in \mathcal{K}} \operatorname{Pr}\left(\mathcal{X}_{\mathrm{MT}_{0}}^{(j, m)}, \mathcal{A}_{\mathrm{MT}_{0}}\right) \bar{F}_{\mathrm{SINR}}\left(\gamma \mid \mathcal{X}_{\mathrm{MT}_{0}}^{(j, m)}, \mathcal{A}_{\mathrm{MT}_{0}}\right) \\
= & \sum_{j \in \mathcal{K}} \sum_{m \in \mathcal{K}} \operatorname{Pr}\left(\mathcal{X}_{\mathrm{MT}_{0}}^{(j, m)}, \mathcal{A}_{\mathrm{MT}_{0}}\right) \times \mathbb{E}_{R_{\mathrm{MT}_{0}}} \mathbb{E}_{I}[\operatorname{Pr} \\
& \left.\left(H_{\mathrm{MT}_{0}}>\frac{\gamma}{p_{0}}\left(I+\sigma_{n}^{2}\right)\left(\tau R_{\mathrm{MT}_{0}}\right)^{\alpha(1-\epsilon)} \mid \mathcal{X}_{\mathrm{MT}_{0}}^{(j, m)}, \mathcal{A}_{\mathrm{MT}_{0}}\right)\right]
\end{aligned}
$$

The proof follows by computing the two remaining expectations.

Corollary 1 Assume $\epsilon=1$, i.e., the active MTs apply a power control scheme based on full channel inversion. The CCDF in Theorem 1 simplifies as follows:

$$
\begin{gathered}
\bar{F}_{S I N R}(\gamma)=\sum_{j \in \mathcal{K}} \sum_{m \in \mathcal{K}} \operatorname{Pr}\left(\mathcal{X}_{M T_{0}}^{(j, m)}, \mathcal{A}_{M T_{0}}\right) \\
\mathrm{e}^{-\gamma \sigma_{n}^{2} / p_{0}} \mathcal{L}_{I}\left(\gamma / p_{0} \mid \mathcal{X}_{M T_{0}}^{(j)}\right)
\end{gathered}
$$

Proof It follows from (21) by setting $\epsilon=1$ and some algebra.

Remark 3 (Operating regimes as a function of $i_{0}$ ) $B y$ direct inspection of Corollary $\mathbf{1}$, three operating regimes as a function of $i_{0}$ can be identified: (i) interference-unaware, where the CCDF of the SINR is independent of $i_{0}$. This occurs if $i_{0}>p_{0}$ and $p_{0} / i_{0}<\min \left(t^{1} / t^{(2)}, t^{(2)} / t^{(1)}\right)$, (ii) interference-aware and cell association independent, where the CCDF of the SINR depends on $i_{0}$ but does not depend on the cell association weights $t^{(1)}$ and $t^{(2)}$. This occurs if $i_{0}<p_{0}$ and $p_{0} / i_{0}>\max \left(t^{(1)} / t^{(2)}, t^{(2)} / t^{(1)}\right)$, (iii) interference-aware and cell association dependent, where the CCDF of the SINR depends on $i_{0}$ and $t^{(\tilde{j})} / t^{(j)}, \forall j \in$ $\mathcal{K}$. This occurs if the conditions above are not satisfied. The same operating regimes can be identified from Propositions 1 and 2. 
Proof It follows by inspection of $\operatorname{Pr}\left(\mathcal{X}_{\mathrm{MT}_{0}}^{(j, m)}, \mathcal{A}_{\mathrm{MT}_{0}}\right)$, $v^{(j)}(v)$ and $\eta^{(j)}(v)$.

The second operating regime, i.e., the performance is independent of the cell association weights, is of particular interest for making the design of HCNs easier: it implies that, for some system parameters, optimizing the DL results in optimizing the UL as well.

It is worth mentioning, in addition, that the conditions that identify the three operating regimes in Remark 3 can be conveniently formulated in decibels as well, which provides further information for system design. More precisely, regime (i) emerges if $i_{0}>p_{0} \mathrm{~dB}$ and $t^{(1)} / t^{(2)} \in$ $\left[-i_{0} / p_{0}, i_{0} / p_{0}\right] \mathrm{dB}$ and regime (ii) emerges if $i_{0}<p_{0} \mathrm{~dB}$ and $t^{(1)} / t^{(2)} \in\left[-p_{0} / i_{0}, p_{0} / i_{0}\right] \mathrm{dB}$.

\section{Smallest path loss association}

In this section, tractable mathematical frameworks under the SPLA scheme are provided. In this case, the condition $t^{(1)}=t^{(2)}$ holds and simplified formulas can be obtained. Under the assumption that the path loss exponents of all the tiers of BSs are the same, in fact, multi-tier $\mathrm{HCNs}$ reduce to an equivalent single-tier cellular network of intensity $\lambda=\sum_{j \in \mathcal{K}} \lambda^{(j)}[2]$.

Proposition 4 The probability that the typical MT is active can be formulated as follows:

$$
\begin{gathered}
\operatorname{Pr}\left(\mathcal{A}_{\mathrm{MT}_{0}}\right)=\int_{r_{1}=0}^{\frac{1}{\tau}\left(\frac{p_{\max }}{p_{0}}\right)^{\frac{1}{\alpha}} 2 \pi \lambda r_{1}} \\
e^{-\pi \lambda \max ^{2}\left(r_{1},\left(\frac{p_{0}}{i_{0}}\right)^{\frac{1}{\alpha}} \frac{\left(\tau r_{1}\right)^{\epsilon}}{\tau}\right)} d r_{1}
\end{gathered}
$$

Proof The proof is similar to that of Proposition 1. The difference is that only the joint PDF of the distance of the nearest and second nearest BSs needs to be used (see Appendix A).

Corollary 2 If $\epsilon=1, \operatorname{Pr}\left(\mathcal{A}_{\mathrm{MT}_{0}}\right)$ in (24) simplifies as follows:

$$
\operatorname{Pr}\left(\mathcal{A}_{\mathrm{MT}_{0}}\right)=\frac{1-\mathrm{e}^{-\frac{\pi}{\tau^{2}}\left(\frac{p_{\max }}{p_{0}}\right)^{\frac{2}{\alpha}} \lambda \max \left(1,\left(\frac{p_{0}}{i_{0}}\right)^{\frac{2}{\alpha}}\right)}}{\max \left(1,\left(\frac{p_{0}}{i_{0}}\right)^{\frac{2}{\alpha}}\right)}
$$

Proof It directly follows from (24) by setting $\epsilon=1$ and computing the integral.

Remark 4 (Operating regimes as a function of $i_{0}$ ) From (25), two operating regimes can be identified: (i) interference-unaware, i.e., $\operatorname{Pr}\left(\mathcal{A}_{\mathrm{MT}_{0}}\right)$ is independent of $i_{0}$, which occurs if $i_{0}>p_{0}$ and (ii) interference-aware, i.e., $\operatorname{Pr}\left(\mathcal{A}_{\mathrm{MT}_{0}}\right)$ depends on $i_{0}$, which occurs if $i_{0}<p_{0}$.

Remark 5 (Unlimited transmit power of the MTs) Assume $p_{\max } \rightarrow \infty$, i.e., the MTs have no maximum transmit power constraint. From (25), the following holds: (i) under the interference-unaware regime $\left(i_{0}>p_{0}\right)$, $\operatorname{Pr}\left(\mathcal{A}_{\mathrm{MT}_{0}}\right) \rightarrow 1$, and (ii) under the interference-aware regime $\left(i_{0}<p_{0}\right), \operatorname{Pr}\left(\mathcal{A}_{\mathrm{MT}_{0}}\right)=\left(i_{0} / p_{0}\right)^{\frac{2}{\alpha}}$. In both regimes, $\operatorname{Pr}\left(\mathcal{A}_{\mathrm{MT}_{0}}\right)$ is independent of the density of BSs $\lambda$.

Lemma 3 The PDF of the distance between the typical $M T$ and its serving $B S$ is as follows:

$$
\begin{aligned}
& f_{R_{\mathrm{MT}_{0}}}\left(v \mid \mathcal{A}_{\mathrm{MT}_{0}}\right)=\frac{2 \pi \lambda v \mathrm{e}^{-\pi \lambda \max ^{2}\left(v,\left(\frac{p_{0}}{i_{0}}\right)^{\frac{1}{\alpha} \frac{(\tau v)^{\epsilon}}{\tau}}\right)}}{\operatorname{Pr}\left(\mathcal{A}_{\mathrm{MT}_{0}}\right)} \\
& \times 1\left(0<v<\frac{1}{\tau}\left(\frac{p_{\max }}{p_{0}}\right)^{\frac{1}{\alpha}}\right)
\end{aligned}
$$

Proof The proof is similar to that of Lemma 1. The difference is that only the joint PDF of the distance of the nearest and second nearest BSs needs to be used (see Appendix A).

Remark 6 (Interference-awareness is equivalent to network densification if $p_{\max } \rightarrow \infty$ ) If the system operates in the interference-aware regime $\left(i_{0}<p_{0}\right)$ and $p_{\max } \rightarrow \infty$, (54) reduces to

$$
f_{R_{\mathrm{MT}_{0}}}\left(v \mid \mathcal{A}_{\mathrm{MT}_{0}}\right)=2 \pi \lambda\left(\frac{p_{0}}{i_{0}}\right)^{\frac{2}{\alpha}} v \mathrm{e}^{-\pi \lambda\left(\frac{p_{0}}{i_{0}}\right)^{\frac{2}{\alpha}} v^{2}}
$$

This implies that IAM's impact is equivalent to increasing the density of BSs from $\lambda$ to $\lambda\left(p_{0} / i_{0}\right)^{\frac{2}{\alpha}}$, since the PDF of the distance from the nearest BS in Poisson cellular networks is $2 \pi \lambda v \mathrm{e}^{-\pi \lambda v^{2}}$. Hence, the distance between probe MT and probe $B S$ is reduced, resulting in better performance.

Proposition 5 If $\epsilon=1$, the average transmit power of the typical MT is as follows:

$$
\begin{gathered}
\mathbb{E}\left[p\left(R_{\mathrm{MT}_{0}}\right)\right]=\frac{p_{0} \tau^{\alpha}}{(\pi \lambda)^{\frac{\alpha}{2}} \max \left(1,\left(\frac{p_{0}}{i_{0}}\right)^{\frac{2}{\alpha}+1}\right)}\left(\Gamma\left(1+\frac{\alpha}{2}\right)\right. \\
\left.-\Gamma\left(\frac{2+\alpha}{2}, \frac{\lambda \pi}{\tau^{2}}\left(\frac{p_{\max }}{p_{0}}\right)^{\frac{2}{\alpha}} \max \left(1,\left(\frac{p_{0}}{i_{0}}\right)^{\frac{2}{\alpha}}\right)\right)\right)
\end{gathered}
$$

Proof If follows from Proposition 2, by setting $\epsilon=1$ and computing the integral. 
Remark 7 (Impact of interference-awareness) If $p_{\max } \rightarrow \infty$ and $i_{0}<p_{0}$ (interference-aware regime), (28) simplifies as follows:

$$
\mathbb{E}\left[p_{\mathrm{MT}}\left(R_{\mathrm{MT}_{0}}\right)\right]=\frac{\tau^{\alpha} \Gamma\left(1+\frac{\alpha}{2}\right)}{(\pi \lambda)^{\frac{\alpha}{2}} p_{0}^{\frac{2}{\alpha}}} i_{0}^{\frac{2}{\alpha}+1}
$$

which implies that the average power consumption of the MTs scales polynomially with exponent $2 / \alpha+1$, as a function of the maximum interference constraint $i_{0}$.

Lemma 4 Assume $\epsilon=1$. The Laplace transform of the aggregate interference can be formulated as $\mathcal{L}_{I}(s)=$ $\exp (\beta(s))$, where $\beta(s)=-2 \pi \lambda \theta \mu(s)$ and the following holds:

$$
\begin{gathered}
\theta=\left(1-\left(1+\frac{\pi}{\tau^{2}} \lambda\left(\frac{p_{\max }}{p_{0}}\right)^{\frac{2}{\alpha}}\right) \times e^{-\frac{\pi}{\tau^{2}} \lambda\left(\frac{p_{\max }}{p_{0}}\right)^{\frac{2}{\alpha}} \max \left(1,\left(\frac{p_{0}}{i_{0}}\right)^{\frac{2}{\alpha}}\right)}\right) \\
\left(\pi \lambda \max \left(1,\left(\frac{p_{0}}{i_{0}}\right)^{\frac{2}{\alpha}}\right)\right)^{-1} \\
\mu(s)=\frac{p_{0} s}{\alpha-2} \max ^{2-\alpha}\left(1,\left(\frac{p_{0}}{i_{0}}\right)^{\frac{1}{\alpha}}\right) \\
\times{ }_{2} \mathrm{~F}_{1}\left(1, \frac{\alpha-2}{\alpha}, 2-\frac{2}{\alpha},-p_{0} s \max ^{-\alpha}\left(1,\left(\frac{p_{0}}{i_{0}}\right)^{\frac{1}{\alpha}}\right)\right)
\end{gathered}
$$

Proof The proof follows from $\chi(s, r)$ in (15), by setting $t^{(1)}=t^{(2)}$ and formulating it as $\chi(s, r)=r^{2} \mu(s)$. Hence, $\beta(s)=-2 \pi \lambda \mu(s) \theta$, where $\theta=\mathbb{E}\left[R_{\mathrm{MT}_{\mathrm{i}}}^{2} \mid A_{\mathrm{MT}_{0}}\right]$.

Proposition 6 Assume $\epsilon=1$. The mean and variance of the interference can be expressed as

$$
\begin{array}{r}
\mathbb{E}[I]=2 \pi \lambda \theta \frac{p_{0} \max ^{2-\alpha}\left(1,\left(\frac{p_{0}}{i_{0}}\right)^{\frac{1}{\alpha}}\right)}{\alpha-2} \\
\operatorname{var}(I)=2 \pi \lambda \theta \frac{p_{0}^{2} \max ^{2-2 \alpha}\left(1,\left(\frac{p_{0}}{i_{0}}\right)^{\frac{1}{\alpha}}\right)}{\alpha-1}
\end{array}
$$

Proof It follows from Lemma 4 evaluating the derivatives of the Laplace transform at zero.

Remark 8 (Trends of mean and variance of the interference as a function of $i_{0}$ ) Assume $p_{\max } \rightarrow \infty$ and consider the interference-aware regime, i.e., $i_{0}<p_{0}$. Then, (30) simplifies to $\theta=\frac{1}{\pi \lambda}\left(\frac{i_{0}}{p_{0}}\right)^{\frac{4}{\alpha}}$ and the mean and variance of the interference can be formulated as follows:

$$
\mathbb{E}[I]=\frac{2}{\alpha-2} p_{0}^{-\frac{2}{\alpha}} i_{0}^{\frac{\alpha+2}{\alpha}} ; \operatorname{var}(I)=\frac{2}{\alpha-1} p_{0}^{-\frac{2}{\alpha}} i_{0}^{\frac{2(\alpha+1)}{\alpha}}
$$

which implies that the mean and variance of the interference scale polynomially with exponents $\alpha+2 / \alpha$ and $2(\alpha+1) / \alpha$ as a function of $i_{0}$, respectively, and they do not depend on the BSs' density.

Finally, the following theorem provides the coverage probability under the SPLA criterion.

Theorem 2 Assume $\epsilon=1, p_{\max } \rightarrow \infty$ and that the system operates in the interference-aware regime $\left(i_{0}<p_{0}\right)$. The CCDF of the SINR can be formulated as follows:

$$
\begin{aligned}
& \bar{F}_{\text {SINR }}\left(\gamma \mid \mathcal{A}_{\mathrm{MT}_{0}}\right)=\exp \left(-\frac{\gamma \sigma_{n}^{2}}{p_{0}}-2 \frac{\gamma}{\alpha-2}\left(\frac{i_{0}}{p_{0}}\right)^{\frac{\alpha+2}{\alpha}}\right. \\
& \left.\quad \times{ }_{2} \mathrm{~F}_{1}\left(1, \frac{\alpha-2}{\alpha}, 2-\frac{2}{\alpha},-\gamma\left(\frac{i_{0}}{p_{0}}\right)\right)\right)
\end{aligned}
$$

Proof The proof follows from Theorem 1 by setting $t^{(1)}=t^{(2)}$ and $\epsilon=1$, and from Lemma 4 by letting $p_{\max } \rightarrow \infty$ and considering $i_{0}<p_{0}$.

Remark 9 (SINR invariance as a function of $\lambda$ ) From (34), we evince that the CCDF of the SINR is independent of $\lambda$, but it depends on the ratio $i_{0} / p_{0}$ and the path loss exponent $\alpha$.

Interestingly, the SINR in such a setup is invariant with the BSs' density. Intuitively, this means that both the desired received power and the interference does not vary with the BSs' density. On the one hand, the desired power does not vary thanks to full channel inversion power control $\left(\epsilon=1, p_{\max } \rightarrow \infty\right)$. Furthermore, although the distances towards the nearest interfering MTs decrease with $\lambda$, their transmit power also decrease with $\lambda$, making the received interference invariant with $\lambda$, as it can be observed from its moments in (33). This density invariance has been also reported in $[2,13]$ for the case of the SIR.

\section{Spectral efficiency and binary rate}

This section is focused on the analysis of SE and BR. Unlike the vast majority of papers on stochastic geometry modeling of $\mathrm{HCNs}$ that evaluate these key performance indicators based on the Shannon formula, we provide a mathematical formulation that is more useful for current cellular deployments based on practical AMC schemes and, thus, provides estimates of SE and BR that can be achieved at a finite target value of the block error rate 
(BLER) rather than their theoretically achievable counterparts under the assumptions of unlimited decoding complexity and arbitrarily small BLER. We show, remarkably, that more tractable expressions of SE and BR can be provided, compared to those that can be obtained based on the Shannon definition. The BR accounts for the amount of bandwidth allocated to the typical MT by the scheduler and, thus, accounts for the BS's load, i.e., the number of MTs that need to be simultaneously served in the cell to which the typical MT belongs to. Accordingly, SE and BR provide information on the advantages and limitations of transmission schemes and, as such, are both employed for assessing the performance of practical LTE systems [14].

$\mathrm{SE}$ and BR, however, are related to each other and, in mathematical terms, we have

$$
\mathrm{BR}_{\mathrm{MT}_{0}}=\frac{b_{w}}{N_{\mathcal{B}_{\mathrm{MT}_{0}}^{A}}^{A}} \mathrm{SE}_{\mathrm{MT}_{0}} \quad \text { (bps) }
$$

where $b_{w}$ is the available bandwidth per BS and $N_{\mathcal{B}_{\mathrm{MT}}}^{\mathcal{A}}$ denotes the number of active MTs associated with the probe BS, which is commonly referred to as the cell load [15].

As extensively discussed in, e.g., [15-17], the distribution of $N_{\mathcal{B}_{\mathrm{MT}}}^{\mathcal{A}}$ is not available for cell association criteria that are not based on the shortest distance, and, thus approximations need to be used. For mathematical tractability, but without loosing in accuracy, we exploit the approximation in [16] which, for the convenience of the readers, is reported in what follows.

Assumption 2 The probability mass function (PMF) of the number of active $M T s, N_{\mathcal{B}_{M T_{0}}}^{\mathcal{A}}$, associated with a BS of tier $j$, is approximated as follows:

$$
\begin{gathered}
\operatorname{Pr}\left(N_{\mathcal{B}_{M T_{0}}^{\mathcal{A}}}^{\mathcal{A}}=n \mid \mathcal{X}_{M T_{0}}^{(j)}, \mathcal{A}_{M T_{0}}\right) \approx \frac{3.5^{3.5}}{(n-1) !} \frac{\Gamma(n+3.5)}{\Gamma(3.5)} \\
\left(\frac{\lambda_{M T} \operatorname{Pr}\left(\mathcal{X}_{M T_{0}}^{(j)}, \mathcal{A}_{M T_{0}}\right)}{\lambda^{(j)}}\right)^{n-1} \\
\left(3.5+\frac{\lambda_{M T} \operatorname{Pr}\left(\mathcal{X}_{M T_{0}}^{(j)}, \mathcal{A}_{M T_{0}}\right)}{\lambda^{(j)}}\right)
\end{gathered}
$$

where, for notational simplicity, the short-hand $p=$ $\operatorname{Pr}\left(\mathcal{X}_{\mathrm{MT}_{0}}^{(j)}, \mathcal{A}_{\mathrm{MT}_{0}}\right)$ is used.

\subsection{Adaptive modulation and coding}

In modern cellular systems [14], AMC is aimed to adapt the MCS to be used to the channel conditions. This is needed for maximizing the BR while providing a BLER below a desired threshold $\mathrm{BLER}_{T}$. In practice, AMC is implemented as follows. In the UL, the MTs transmit sounding reference signals that are used by the BSs for estimating the SINR. Based on these estimates, the BSs choose the MCS to use (usually identified by an index), which corresponds to a given Channel Quality Indicator (CQI), $i_{\mathrm{CQI}} \in\left[1, n_{\mathrm{CQI}}\right]$, that maximizes the SE while maintaining the BLER below BLER ${ }_{T}$. The choice of the best MCS to use is made based on lookup tables that provide the SINR thresholds, $\gamma_{i_{\mathrm{COI}}}$, associated to each value of CQI. Finally, the BSs inform each scheduled MT of the MCS index to use for its subsequent transmission. To reduce the reporting overhead associated with the CQIs, the LTE standard assumes that the number of bits used for reporting the CQI is equal to 4 , which implies $n_{\mathrm{CQI}}=15$.

Based on this working principle, the BR can be obtained from (35) and the SE is as follows:

$$
\mathrm{SE}_{\mathrm{MT}_{0}}=\sum_{i_{\mathrm{CQI}}=1}^{n_{\mathrm{CQI}}} \mathrm{SE}_{i_{\mathrm{CQI}}} \mathbf{1}\left(\mathrm{SINR}_{\mathrm{MT}_{0}} \in\left[\gamma_{i_{\mathrm{CQI}}}, \gamma_{i_{\mathrm{CQI}+1}}\right)\right)
$$

where $\gamma_{1}<\cdots<\gamma_{n_{\mathrm{CQI}}}, i_{\mathrm{CQI}}=0$ if no transmission, $\bigcap_{i_{\mathrm{CQI}}=1}^{n_{\mathrm{CQI}}}\left[\gamma_{i_{\mathrm{CQI}}}, \gamma_{i_{\mathrm{CQI}+1}}\right)=\emptyset, \gamma_{n_{\mathrm{CQI}+1}} \rightarrow \infty$.

Based on (37), the spatially average SE can be obtained from the CCDF of the SINR provided in previous sections for GCA and SPLA criteria, respectively. More precisely, we have

$$
\begin{aligned}
\mathbb{E} & {\left[\mathrm{SE}_{\mathrm{MT}_{0}}\right]=\sum_{j \in \mathcal{K}} \sum_{m \in \mathcal{K}} \operatorname{Pr}\left(\mathcal{X}_{\mathrm{MT}_{0}}^{(j, m)}, \mathcal{A}_{\mathrm{MT}_{0}}\right) } \\
& \sum_{i_{\mathrm{CQI}}=1}^{n_{\mathrm{CQI}}} \mathrm{SE}_{i_{\mathrm{CQI}}}\left(\bar{F}_{\mathrm{SINR}}\left(\gamma_{i_{\mathrm{CQI}}} \mid \mathcal{X}_{\mathrm{MT}_{0}}^{(j, m)}, \mathcal{A}_{\mathrm{MT}_{0}}\right)\right. \\
& \left.-\bar{F}_{\mathrm{SINR}}\left(\gamma_{i_{\mathrm{CQI}}+1} \mid \mathcal{X}_{\mathrm{MT}_{0}}^{(j, m)}, \mathcal{A}_{\mathrm{MT}_{0}}\right)\right)
\end{aligned}
$$

With similar arguments, the average BR of the probe MT can be written as follows:

$$
\begin{aligned}
\mathbb{E} & {\left[\mathrm{BR}_{\mathrm{MT}_{0}}\right]=\sum_{j \in \mathcal{K}} \sum_{m \in \mathcal{K}} \sum_{n>0} \operatorname{Pr}\left(\mathcal{X}_{\mathrm{MT}_{0}}^{(j, m)}, \mathcal{A}_{\mathrm{MT}_{0}}\right) } \\
& \operatorname{Pr}\left(N_{\mathcal{B}_{\mathrm{MT}}}^{\mathcal{A}}=n \mid \mathcal{X}_{\mathrm{MT}_{0}}^{(j)}, \mathcal{A}_{\mathrm{MT}_{0}}\right) \\
& \sum_{i_{\mathrm{CQI}}=1}^{n_{\mathrm{CQI}}} \frac{b_{w}}{n} \mathrm{SE}_{i_{\mathrm{CQI}}}\left(\bar{F}_{\mathrm{SINR}}\left(\gamma_{i_{\mathrm{CQI}}} \mid \mathcal{X}_{\mathrm{MT}_{0}}^{(j, m)}, \mathcal{A}_{\mathrm{MT}_{0}}\right)\right. \\
& \left.-\bar{F}_{\mathrm{SINR}}\left(\gamma_{i_{\mathrm{CQI}}+1} \mid \mathcal{X}_{\mathrm{MT}_{0}}^{(j, m)}, \mathcal{A}_{\mathrm{MT}_{0}}\right)\right) \\
& \stackrel{(\mathrm{a})}{=} \sum_{j \in \mathcal{K}} \sum_{m \in \mathcal{K}} \sum_{i_{\mathrm{CQI}}=1}^{n_{\mathrm{CQI}}} \operatorname{Pr}\left(\mathcal{X}_{\mathrm{MT}_{0}}^{(j, m)}, \mathcal{A}_{\mathrm{MT}_{0}}\right)
\end{aligned}
$$




$$
\begin{array}{r}
\times \mathrm{SE}_{i_{\mathrm{CQI}}} \frac{3.5^{3.5} b_{w}\left(3.5 \lambda^{(j)}+\lambda_{\mathrm{MT}} p\right)}{\lambda_{\mathrm{MT}} p\left(1-\frac{\lambda_{\mathrm{MT}} p}{\lambda^{(j)}}\right)^{3.5}} \\
\times\left(1-\left(1-\frac{\lambda_{\mathrm{MT} p}}{\lambda^{(j)}}\right)^{3.5}\right) \\
\times\left(\bar{F}_{\mathrm{SINR}}\left(\gamma_{i_{\mathrm{CQI}}} \mid \mathcal{X}_{\mathrm{MT}_{0}}^{(j, m)}, \mathcal{A}_{\mathrm{MT}_{0}}\right)\right. \\
\left.-\bar{F}_{\mathrm{SINR}}\left(\gamma_{i_{\mathrm{CQI}}+1} \mid \mathcal{X}_{\mathrm{MT}_{0}}^{(j, m)}, \mathcal{A}_{\mathrm{MT}_{0}}\right)\right)
\end{array}
$$

where (a) is obtained by computing the summation over $n=N_{\mathcal{B}_{\mathrm{MT}}}^{\mathcal{A}}$ in closed-form with the aid of the PMF in (36).

The mathematical expressions of SE and BR of AMC schemes are easier to compute than the corresponding formulas obtained from the Shannon definition of SE, since the latter definition requires an extra integral to be computed [15]. This is remarkable, since the SE and BR in (38) and (39) account for feedback's overhead and limited-complexity receivers.

In the present paper, as a sensible case study, we consider the range of CQI values and a target BLER equal to $10 \%$, as recommended by LTE specifications [14]. The SINR thresholds $\gamma_{i_{\mathrm{CQI}}}$ are obtained from link-level simulations conducted with an accurate LTE simulator $[9,10]$. More precisely, the considered simulator assumes MTs of limited computational complexity, where decoding is performed by using a 1-tap zero forcing equalizer and a turbo decoder based on the soft output Viterbi algorithm. Numerical illustrations are reported in Section 8. For completeness, Table 2 reports the input parameters that are needed for computing the SE and BR in (38) and (39). It is worth emphasizing, however, that (38) and (39) are general enough for being used for analyzing different wireless standards and receiver implementations.

\section{On fairness-a hybrid muting scheme}

In this section, we introduce a new hybrid interferenceaware muting scheme in order to overcome some of the limitations of the IAM scheme, especially in terms of fairness among the MTs. Due to the specific operations of the IAM scheme, some MTs may be muted for long time, which is unfair for them compared to the MTs that are scheduled for transmission. To overcome this limitation, we propose a transmission scheme whose operation is split into two time slots. In time slot 1, the IAM is applied as described in the previous sections. This implies that some MTs are muted and do not transmit. In time slot 2 , the MTs that are muted during the first time slot are the only ones allowed to transmit signals. The rationale for this scheme is that the first time slot is optimized for transmission based on the IAM scheme, while the second time slot allow us to avoid unfairness among the MTs. The duration of each time slot is an optimization variable, which allows us to identify the best transmission scheme to use. If the duration of the first time slot is $100 \%$ of the entire time allocated for transmission, then the hybrid scheme boils down to the IAM scheme. If, on the other hand, the duration of the second time slot is $100 \%$ of the entire time allocated for transmission, then the hybrid scheme boils down to the conventional scheme where all the MTs are allowed to transmit, which avoids fairness issues. The optimization of the duration of each time slot, given the total time allocated for transmission, is an important parameter to trade-off performance and fairness. In this section, we analyze the performance of this hybrid scheme.

The average coverage probability, SE and BR for the hybrid scheme can be formulated, respectively, as follows:

$$
\begin{array}{r}
\mathbb{E}\left[\bar{F}_{\mathrm{SINR}}(\gamma)\right]=t_{1} \bar{F}_{\mathrm{SINR}}(\gamma)+\left(1-t_{1}\right) \overline{\bar{F}_{\mathrm{SINR}}}(\gamma) \\
\mathbb{E}\left[\mathrm{SE}_{\mathrm{MT}_{0}}\right]=t_{1} \mathrm{SE}_{\mathrm{MT}_{0}}+\left(1-t_{1}\right) \overline{\mathrm{SE}_{\mathrm{MT}_{0}}} \\
\mathbb{E}\left[\mathrm{BR}_{\mathrm{MT}_{0}}\right]=t_{1} \mathrm{BR}_{\mathrm{MT}_{0}}+\left(1-t_{1}\right) \overline{\mathrm{BR}_{\mathrm{MT}_{0}}}
\end{array}
$$

where $t_{1}$ denotes the duration of the first time slot, and the entire time allocated for transmission is normalized to 1 for simplicity. This implies that the duration of the second time slot is $1-t_{1}$. The symbols with the "overline" denote the performance metrics during the second time slots, where the MTs that are muted in the first time slot are the only ones allowed to transmit.

Since the network operation during the first time slot coincides with the IAM scheme that is studied in the previous sections, in this section, we analyze only the performance during the second time slot. In this case, we

\begin{tabular}{|c|c|c|c|c|c|c|c|c|c|c|c|c|c|c|c|}
\hline$i_{\mathrm{CQI}}$ & 1 & 2 & 3 & 4 & 5 & 6 & 7 & 8 & 9 & 10 & 11 & 12 & 13 & 14 & 15 \\
\hline $\mathrm{SE}_{i_{\mathrm{CQI}}}[\mathrm{bps} / \mathrm{Hz}]$ & 0.15 & 0.23 & 0.38 & 0.60 & 0.88 & 1.18 & 1.48 & 1.91 & 2.41 & 2.73 & 3.32 & 3.90 & 4.52 & 5.11 & 5.55 \\
\hline$\gamma_{i_{\mathrm{CQI}}}[\mathrm{dB}]$ & -3.65 & -1.60 & 0.00 & 2.25 & 3.75 & 4.75 & 9.00 & 10.50 & 12.35 & 15.40 & 17.18 & 18.85 & 20.70 & 24.0 & 25.0 \\
\hline
\end{tabular}
need to replace $\mathcal{A}_{\mathrm{MT}_{i}}$ with $\overline{\mathcal{A}_{\mathrm{MT}}}$, based on the notation in Table 1.

\subsection{General cell association criterion}

We start introducing some enabling results to prove the main theorems.

Table 2 SINR thresholds and SE values obtained from the LTE link-level simulator in $[9,10]$ 
Proposition 7 The probability that the typical MT is muted and is associated with tier $j$ is

$$
\begin{aligned}
& \operatorname{Pr}\left(\overline{\mathcal{A}_{M T_{0}}}\right)=\sum_{j \in \mathcal{K}_{v>0}}\left(\omega^{(j)}(v)+\zeta^{(j)}(v)\right) \mathrm{d} v \\
& -\sum_{j \in \mathcal{K}_{v>0}} \mathbf{1}\left(v<\frac{1}{\tau}\left(\frac{p_{\max }}{p_{0}}\right)^{\frac{1}{\alpha}}\right) \\
& \times\left(v^{(j)}(v)+\eta^{(j)}(v)\right) \mathrm{d} v
\end{aligned}
$$

where $v^{(j)}(v), \eta^{(j)}(v), \omega^{(j)}(v)$, and $\zeta^{(j)}(v)$ are defined in $(9)$, (10), (45), and (46), respectively.

$$
\begin{aligned}
& \omega^{(j)}(v)=2 \pi \lambda^{(j)} v\left(\mathrm{e}^{-\pi \lambda^{(j)} v^{2}}\left(\mathrm{e}^{-\pi \lambda^{\widetilde{j})}\left(\left(\frac{t^{(\widetilde{j})}}{t^{(j)}}\right)^{\frac{1}{\alpha}} v\right)^{2}}-\mathrm{e}^{-\pi \lambda(\widetilde{j}) v^{2}}\right)\right. \\
& \times 1\left(\left(\frac{t^{(\widetilde{j})}}{t^{(j)}}\right)^{\frac{1}{\alpha}}<1\right)+\frac{\lambda^{\widetilde{j})}}{\lambda^{(\widetilde{j})}+\lambda^{(j)}} \mathrm{e}^{\left.-\pi\left(\lambda^{\widetilde{j})}+\lambda^{(j)}\right) \max ^{2}\left\{\left(\frac{t^{(\widetilde{j})}}{t^{(j)}}\right)^{\frac{1}{\alpha}} v\right\}\right)}
\end{aligned}
$$

$$
\begin{aligned}
\zeta^{(j)}(v) & =2 \pi \lambda^{(j)} v\left(\mathrm{e}^{-\pi \lambda^{(j)} v^{2}} \mathrm{e}^{-\pi \lambda^{(j)} \max ^{2}\left\{\left(\frac{t^{(j)}}{t^{(j)}}\right)^{\frac{1}{\alpha}} v, v\right\}}\right. \\
& \left.-\frac{\lambda^{(\widetilde{j})}}{\lambda^{(\widetilde{j})}+\lambda^{(j)}} \mathrm{e}^{-\pi\left(\lambda^{(j)}+\lambda^{(j)}\right) \max ^{2}\left\{\left(\frac{t^{(j)}}{t^{(j)}}\right)^{\frac{1}{\alpha}} v, v\right\}}\right)
\end{aligned}
$$

Proof See Appendix C.

Lemma 5 The probability density function (PDF) of the distance between the typical MT and its serving BS by conditioning on the event $\mathcal{X}_{M T_{0}}^{(j, m)} \cap \overline{\mathcal{A}_{M T_{0}}}$ can be formulated as follows:

$$
\begin{aligned}
& f_{R_{M T_{0}}}\left(v \mid \mathcal{X}_{M T_{0}}^{(j, m)}, \overline{\mathcal{A}_{M T_{0}}}\right)= \\
& \begin{cases}\frac{\omega^{(j)}(v)-v^{(j)}(v) \mathbf{1}\left(v<\frac{1}{\tau}\left(\frac{p_{\max }}{p_{0}}\right)^{\frac{1}{\alpha}}\right)}{\operatorname{Pr}\left(\mathcal{X}_{M T_{0}}^{(j, m)}, \overline{\mathcal{A}_{M T_{0}}^{(j, m)}}\right)} & \text { if } j \neq m \\
\frac{\zeta^{(j)}(v)-\eta^{(j)}(v) \mathbf{1}\left(v<\frac{1}{\tau}\left(\frac{p_{\max }}{p_{0}}\right)^{\frac{1}{\alpha}}\right)}{\operatorname{Pr}\left(\mathcal{X}_{M T_{0}}^{(j, j)}, \overline{\mathcal{A}_{M T_{0}}^{(j, j)}}\right)} & \text { if } j=m\end{cases}
\end{aligned}
$$

where $v^{(j)}(v), \eta^{(j)}(v), \omega^{(j)}(v)$, and $\zeta^{(j)}(v)$ are defined in $(9)$, (10), (45), and (46) respectively.

Proof The Cumulative Distribution Function (CDF) of the distance between the typical MT and its serving BS by conditioning on the MT being muted and on $\mathcal{X}_{\mathrm{MT}_{0}}^{(j, m)}$ is obtained by using steps similar to Appendix A. The PDF is obtained from the CDF by computing the derivative.

Lemma 6 Assume that the typical MT is associated with a BS of tier $j$. The Laplace transform of the (conditional) interference in time slot 2 can be formulated as follows:

$$
\mathcal{L}_{I M}\left(s \mid \mathcal{X}_{M T_{0}}^{(j)}\right)=\exp \left(\beta_{\text {all }}^{(j)}(s)-\beta^{(j)}(s)\right)
$$

where $\beta^{(j)}(s)$ is defined in Lemma 2 and $\beta_{\text {all }}^{(j)}(s)$ is defined as follows:

$$
\begin{array}{r}
\beta_{\text {all }}^{(j)}(s)=-\sum_{k \in \mathcal{K}} 2 \pi \lambda^{(k)} \sum_{n \in \mathcal{K}} \operatorname{Pr}\left(\mathcal{Q}_{M T_{i}}^{(n)} \mid \mathcal{X}_{M T_{i}}^{(k, n)}\right) \\
\int_{0}^{\infty} f_{R_{M T_{i}}}\left(r \mid \mathcal{X}_{M T_{i}}^{(j, m)}\right) \chi_{\text {all }}(s, r) d r
\end{array}
$$

where $f_{R_{M T_{i}}}\left(r \mid \mathcal{X}_{M T_{i}}^{(k, n)}\right)$ is the PDF of the distance between the ith interfering MT and its serving BS for all the MTs, $\chi_{\text {all }}(s, r)$ is defined as follows:

$$
\begin{aligned}
& \chi_{\mathrm{all}}(s, r)=\frac{s(\tau)^{-\alpha}(\tau r)^{\alpha \varepsilon} p_{0}}{\alpha-2} r^{2-\alpha} \\
&{ }_{2} F_{1}\left[1, \frac{\alpha-2}{\alpha}, 2-\frac{2}{\alpha},-r^{-\alpha} s(\tau)^{-\alpha}(\tau r)^{\alpha \varepsilon} p_{0}\right] d r
\end{aligned}
$$

and $\operatorname{Pr}\left(\mathcal{Q}_{M T_{i}}^{(n)} \mid \mathcal{X}_{M T_{i}}^{(k)}\right)$ is defined as follows:

$$
\begin{aligned}
& \operatorname{Pr}\left(\mathcal{Q}_{M T_{i}}^{(n)} \mid \mathcal{X}_{M T_{i}}^{(k)}\right)=\frac{\operatorname{Pr}\left(\mathcal{X}_{M T_{i}}^{(k, n)}\right)}{\operatorname{Pr}\left(\mathcal{X}_{M T_{i}}^{(k)}\right)} \\
& =\frac{\operatorname{Pr}\left(\mathcal{X}_{M T_{i}}^{(k, n)}\right)}{\sum_{q \in \mathcal{K}} \operatorname{Pr}\left(\mathcal{X}_{M T_{i}}^{(k, q)}\right)}
\end{aligned}
$$


Theorem 3 The CCDF of the SINR of the typical MT in time slot 2 can be formulated as follows:

$$
\begin{aligned}
& \overline{\overline{F_{S I N R}}}(\gamma)=\sum_{j \in \mathcal{K}} \sum_{m \in \mathcal{K}} \operatorname{Pr}\left(\mathcal{X}_{M T_{0}}^{(j, m)}, \overline{\mathcal{A}_{M T_{0}}}\right) \\
& \int_{\nu=0}^{\infty} f_{R_{M T_{0}}}\left(v \mid \mathcal{X}_{M T_{0}}^{(j, m)}, \overline{\mathcal{A}_{M T_{0}}}\right) \\
& \mathrm{e}^{-\gamma \sigma_{n}^{2}(\tau \nu)^{\alpha(1-\epsilon)}} p_{0}^{-1} \mathcal{L}_{I M}\left(\gamma(\tau \nu)^{\alpha(1-\epsilon)} p_{0}^{-1} \mid \mathcal{X}_{M T_{0}}^{(j)}\right) \mathrm{d} v
\end{aligned}
$$

\subsection{Smallest path loss association}

In this section, we study the smallest path loss cell association criterion. In this case, the condition $t^{(1)}=t^{(2)}$ holds and simplified formulas can be obtained. Under the assumption that the path loss exponents of all the tiers of BSs are the same, in fact, multi-tier HCNs reduce to an equivalent single-tier cellular network of intensity $\lambda=$ $\sum_{j \in \mathcal{K}} \lambda^{(j)}[2]$.

Proposition 8 The probability that the typical MT is muted can be formulated as follows:

$$
\begin{aligned}
& \operatorname{Pr}\left(\overline{\mathcal{A}_{M T_{0}}}\right)=\int_{0}^{\infty} 2 \pi \lambda r_{1} \mathrm{e}^{-\pi \lambda r_{1}^{2}}-2 \pi \lambda r_{1} \\
& \left.\times \mathrm{e}^{-\pi \lambda \max ^{2}\left\{r_{1},\left(\frac{p_{0}}{i_{0}}\right)^{\frac{1}{\alpha}} \frac{\left(\tau r_{1}\right)^{\varepsilon}}{\tau}\right\}}\right\}_{1}\left(r_{1}<\frac{1}{\tau}\left(\frac{p_{\max }}{p_{0}}\right)^{\frac{1}{\alpha \varepsilon}}\right) d r_{1}
\end{aligned}
$$

Proof The proof is similar to that of Proposition 7.

Corollary 3 If $\epsilon=1, \operatorname{Pr}\left(\mathcal{A}_{\mathrm{MT}_{0}}\right)$ in (52) simplifies as follows:

$$
\operatorname{Pr}\left(\overline{\mathcal{A}_{\mathrm{MT}_{0}}}\right)=1-\frac{1-\mathrm{e}^{-\frac{\pi}{\tau^{2}}\left(\frac{p_{\max }}{p_{0}}\right)^{\frac{2}{\alpha}} \lambda \max \left(1,\left(\frac{p_{0}}{i_{0}}\right)^{\frac{2}{\alpha}}\right)}}{\max \left(1,\left(\frac{p_{0}}{i_{0}}\right)^{\frac{2}{\alpha}}\right)}
$$

Proof It directly follows from 52 by setting $\epsilon=1$ and computing the integral.

Lemma 7 The PDF of the distance between the typical $M T$ and its serving $B S$ is as follows:

$$
\begin{aligned}
& f_{R_{M T_{0}}}\left(v \mid \overline{\mathcal{A}_{M T_{0}}}\right)=\frac{2 \pi \lambda \nu \mathrm{e}^{-\pi \lambda v^{2}}}{\operatorname{Pr}\left(\overline{\mathcal{A}_{M T_{0}}}\right)} \\
& -\frac{\left.2 \pi \lambda v \mathrm{e}^{-\pi \lambda \max ^{2}\left\{v\left(\frac{p_{0}}{i_{0}}\right)^{\frac{1}{\alpha}} \frac{\left(\tau \nu v^{\varepsilon}\right.}{\tau}\right.}\right\}_{1}\left(v<\frac{1}{\tau}\left(\frac{p_{\max }}{p_{0}}\right)^{\frac{1}{\alpha \varepsilon}}\right)}{\operatorname{Pr}\left(\overline{\mathcal{A}_{M T_{0}}}\right)}
\end{aligned}
$$

Proof The proof is similar to that of Lemma 5. The difference is that only the joint PDF of the distance of nearest and second nearest BSs needs to be used (see Appendix C).

Lemma 8 Assume $\epsilon=1$. The Laplace transform of the aggregate interference can be formulated as $\mathcal{L}_{I}(s)=$ $\exp \left(\beta_{\mathrm{all}}(s)-\beta(s)\right)$, where $\beta(s)$ is defined in Lemma 4 and $\beta_{\text {all }}(s)$ can be denoted as following:

$$
\beta_{\text {all }}(s)=\frac{-2 s p_{0}}{\alpha-2}{ }_{2} F_{1}\left[1, \frac{\alpha-2}{\alpha}, 2-\frac{2}{\alpha},-s p_{0}\right]
$$

Proof The proof follows from $\chi_{\text {all }}(s, r)$ in (49), by setting $t^{(1)}=t^{(2)}$ and computing the integral.

Theorem 4 Assume $\epsilon=1, p_{\max } \rightarrow \infty$ and that the system operates in the interference-aware regime $\left(i_{0}<p_{0}\right)$. The CCDF of the SINR in time slot 2 can be formulated as follows:

$$
\begin{aligned}
& \overline{\bar{F}_{\text {SINR }}}(\gamma)=\exp \left(-\frac{\gamma}{p_{0}} \sigma_{n}^{2}-\frac{2 \gamma}{\alpha-2} 2 F_{1}\left[1, \frac{\alpha-2}{\alpha}, 2-\frac{2}{\alpha},-\gamma\right]\right) \\
& \times \exp \left(\frac{2 \gamma}{\alpha-2}\left(\frac{i_{0}}{p_{0}}\right)^{\frac{\alpha+2}{\alpha}}{ }_{2} F_{1}\left(1, \frac{\alpha-2}{\alpha}, 2-\frac{2}{\alpha},-\gamma \frac{i_{0}}{p_{0}}\right)\right)
\end{aligned}
$$

Proof With the aid of the total probability theorem, we have:

$$
\begin{array}{r}
\overline{\overline{\bar{F}_{\mathrm{SINR}}}}(\gamma)=0 \times \operatorname{Pr}\left(\mathcal{A}_{\mathrm{MT}_{0}}\right)+\overline{\overline{F_{\mathrm{SINR}}}}\left(\gamma \mid \overline{\mathcal{A}_{\mathrm{MT}_{0}}}\right) \operatorname{Pr}\left(\overline{\mathcal{A}_{\mathrm{MT}_{0}}}\right) \\
=\sum_{j \in \mathcal{K}} \sum_{m \in \mathcal{K}} \operatorname{Pr}\left(\mathcal{X}_{\mathrm{MT}_{0}}^{(j, m)}, \overline{\mathcal{A}_{\mathrm{MT}_{0}}}\right) \overline{\bar{F}_{\mathrm{SINR}}}\left(\gamma \mid \mathcal{X}_{\mathrm{MT}_{0}}^{(j, m)}, \overline{\mathcal{A}_{\mathrm{MT}_{0}}}\right) \\
=\sum_{j \in \mathcal{K}} \sum_{m \in \mathcal{K}} \operatorname{Pr}\left(\mathcal{X}_{\mathrm{MT}_{0}}^{(j, m)}, \overline{\mathcal{A}_{\mathrm{MT}_{0}}}\right) \times \mathbb{E}_{R_{\mathrm{MT}_{0}}} \mathbb{E}_{I}[\operatorname{Pr} \\
\left.\left(H_{\mathrm{MT}_{0}}>\frac{\gamma}{p_{0}}\left(I+\sigma_{n}^{2}\right)\left(\tau R_{\mathrm{MT}_{0}}\right)^{\alpha(1-\epsilon)} \mid \mathcal{X}_{\mathrm{MT}_{0}}^{(j, m)}, \overline{\mathcal{A}_{\mathrm{MT}_{0}}}\right)\right]
\end{array}
$$

The proof follows by computing the two remaining expectations.

\section{Numerical results and discussion}

In this section, we validate the mathematical frameworks and findings derived in the previous sections with the aid of Monte Carlo simulations, as well as compare the IAM scheme against IAFPC and IUFPC schemes. The following setup compliant with LTE specifications is considered. The bandwidth is equal to $10 \mathrm{MHz}$, which implies $b_{w}=9 \mathrm{MHz}$ by excluding the guard bands. The noise power spectral density is $n_{\text {thermal }}=-174 \mathrm{dBm} / \mathrm{Hz}$, and the noise figure of the receiver is $n_{\mathrm{F}}=9 \mathrm{~dB}$. Both GCA and SPLA criteria are studied, and the association weights are, unless otherwise stated, $t^{(1)} / t^{(2)}=$ $9 \mathrm{~dB}$ and $t^{(1)} / t^{(2)}=0 \mathrm{~dB}$, respectively. The case study 
$t^{(1)} / t^{(2)}=9 \mathrm{~dB}$ is related to a cell association based on the average DL received power criterion, where the first tier of BSs (macro) has transmit power equal to $46 \mathrm{dBm}$ and the second tier of BSs (small-cell) has transmit power equal to $37 \mathrm{dBm}$, which agrees with ([18], Annex A: Simulation Model). Other simulation parameters are provided in Table 3. As far as Monte Carlo simulations are concerned, they are obtained by considering $10^{4}$ realizations of channels and network topologies. In all the figures, analytical and Monte Carlo simulation results are represented with solid lines and markers, respectively.

\subsection{Average transmit power, probability of being active, mean, and variance of the interference}

In this section, we analyze the average transmit power of the MTs, the probability that the typical MT is active, which provides information on the system fairness, and the mean and variance of the interference.

Figures 1, 2, 3, and 4 confirm the conclusions drawn in Remark 1, i.e., the mathematical frameworks of average transmit power and probability of being active are exact while those of mean and variance of the interference are approximations that exploit Assumption 1. Such an assumption considers that the position of interfering MTs can be modeled as a conditionally thinned (i.e., nonhomogeneous) PPP. The difference between such a nonhomogeneous PPP, and the actual point process, which is on the other hand not tractable, explains also the difference between simulation and analytical results in all the metrics that depend on the interference (SINR, SE, BR). The conclusions drawn in Remark 2 are confirmed as well: the mean and variance of the interference decrease by decreasing $i_{0}$, which provide important advantages for implementing AMC schemes.

In the figures, IAM and IAFPC are compared as well. We observe that IAM reduces the average transmit power and the mean and variance of the interference.

Consider the SPLA criterion, which is illustrated with dashed lines in the figures. We observe that the findings in Remark 4 are confirmed: the system is interferenceaware and interference-unaware if $i_{0}<p_{0}$ and $i_{0}>p_{0}$, respectively. As expected, the crossing point occurs at

Table 3 Simulation setup

\begin{tabular}{llll}
\hline Parameter & Value & Parameter & Value \\
\hline$f_{C}(\mathrm{MHz})$ & $2 \times 10^{3}$ & $h_{\mathrm{BS}}(\mathrm{m})$ & 10 \\
$b_{W}(\mathrm{MHz})$ & 9 & $t^{(1)} / t^{(2)}(\mathrm{dB})$ & $\{9,0\}$ \\
$\lambda^{(1)}\left(\right.$ points $\left./ \mathrm{m}^{2}\right)$ & $2 \times 10^{-6}$ & $\lambda^{(2)}\left(\right.$ points $\left./ \mathrm{m}^{2}\right)$ & $4 \times 10^{-6}$ \\
$\lambda_{\mathrm{MT}}\left(\right.$ points $\left./ \mathrm{m}^{2}\right)$ & $80 \times 10^{-6}$ & $n_{\text {thermal }}(\mathrm{dBm} / \mathrm{Hz})$ & -174 \\
$n_{\mathrm{F}}(\mathrm{dB})$ & 9 & $\sigma_{s}(\mathrm{~dB})$ & 4 \\
$p_{0}(\mathrm{dBm})$ & -70 & $p_{\max }(\mathrm{dBm})$ & $\{\infty, 5\}$ \\
$i_{0}(\mathrm{dBm})$ & {$[-120,-60]$} & $\epsilon$ & {$[0,1]$} \\
\hline
\end{tabular}

$p_{0}=-70 \mathrm{dBm}$ based on the simulation parameters used. In addition, the scaling laws of average transmit power and average interference are in agreement with the findings in Remark 7 and Remark 8.

All in all, the numerical illustrations reported in Figs. 1, 2, 3, and 4 confirm all the conclusions and performance trends discussed in the previous sections and highlight the advantages of IAM.

\subsection{Complementary cumulative distribution function of the SINR}

In this section, we analyze the coverage probability (CCDF of the SINR) of the active MTs. The results are illustrated in Figs. 5 and 6 for $\epsilon=1$ and $\epsilon=0.75$, respectively, and by assuming $p_{\max } \rightarrow \infty$.

In both figures, we observe a good agreement between mathematical frameworks and Monte Carlo simulations. In particular, the figures confirm, once again, that the coverage probability of IAM increases as $i_{0}$ decreases. In Fig. 5, for example, almost all the active MTs have a SINR greater than $20 \mathrm{~dB}$ if $i_{0}=-120 \mathrm{dBm}$. This good SINR is obtained because IAM keeps under control the interference by muting the MTs that create more interference. Based on Fig. 2, in fact, we note that only a small fraction of the MTs are allowed to be active for $i_{0}=-120 \mathrm{dBm}$. The active MTs, however, better exploit the available bandwidth. Similar conclusions can be drawn for $\epsilon=0.75$ shown in Fig. 6 . The main difference is that, in this latter figure, IAM provides almost the same coverage probability for $i_{0}=-60 \mathrm{dBm}$ and $i_{0}=-90$ $\mathrm{dBm}$. The reason is that the MTs transmit with less power if $\epsilon=0.75$ and, thus, there is almost no difference between the two interference constraints. This brings to our attention that the design of the UL of HCNs requires to jointly optimize $i_{0}, p_{0}, p_{\max }$, and $\epsilon$, in order to identify the desired operating regime that fulfills the requirements in terms of system fairness and interference mitigation. The proposed mathematical frameworks can be used to this end.

\subsection{Spectral efficiency and binary rate}

In this section, the average $\mathrm{SE}$ and average $\mathrm{BR}$ are analyzed, as well as the IAFPC and IAM schemes are compared against each other for several system setups.

In Fig. 7, the average SE of IAFPC and IAM schemes is analyzed and three conclusions can be drawn. By comparing the average SE of the IAPFC scheme (i.e., for AMC schemes) and on the Shannon formula, we note, as expected, that the latter formula provides optimistic estimates of the average SE. By comparing the average SE of the IAM scheme for typical (active and muted) MTs and active (only) MTs, we note a different performance trend as a function of $i_{0}$. As for the active MTs, the average SE increases as $i_{0}$ decreases. As for the typical MTs, on the 


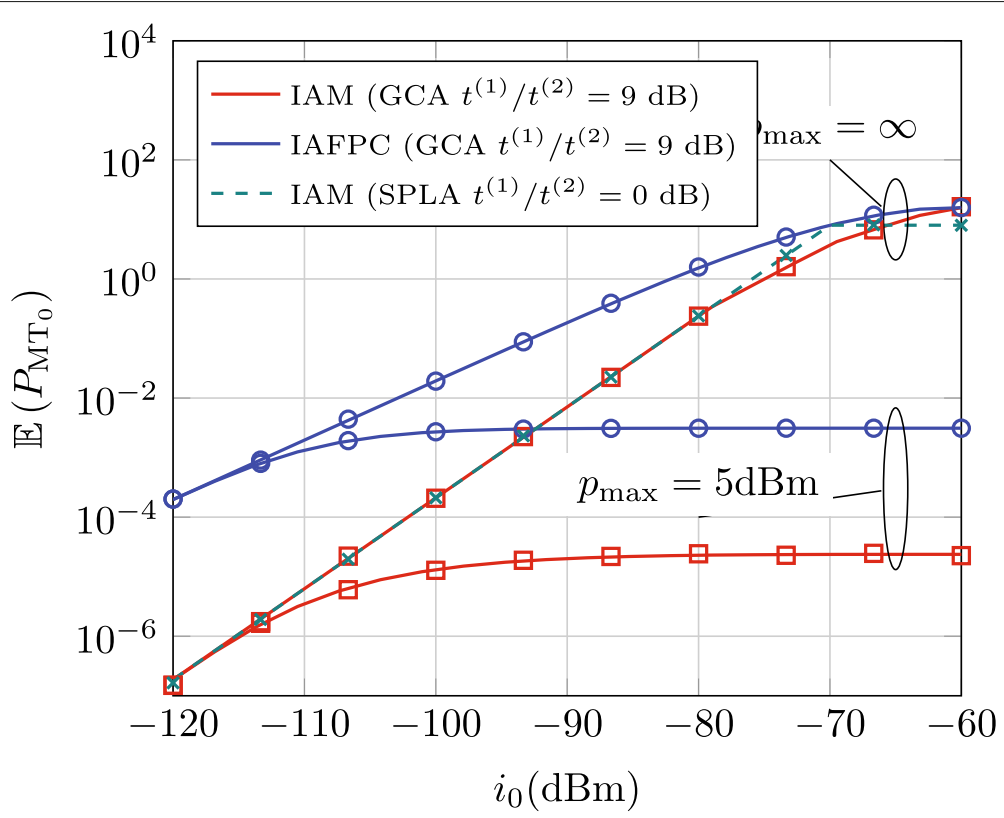

Fig. 1 Average transmit power versus $i_{0}$ for IAM and IAFPC methods with $\epsilon=1, p_{\max } \rightarrow \infty$, and $p_{\max }=5 \mathrm{dBm}$

other hand, the average SE decreases as $i_{0}$ decreases. This is because the lower $i_{0}$ is the more MTs are turned off, which on average, contributes to reduce the SE of the typical MT. By comparing the average SE of IAPFC and IAM schemes, we evince that IAFPC outperforms IAM for all relevant values of the maximum interference constraint $i_{0}$, since all the MTs are active under the IAFPC scheme. The average SE of the active MTs under the IAM scheme is, however, much better than that of the IAFPC scheme, since the other-cell interference is reduced.

The SE, however, does not provide information on the amount of bandwidth that the scheduler allocates to each active MT.

This trade-off is captured by the average BR, which is shown Fig. 8. As far as the average BR is concerned, in particular, we note that IAFPC and IAM schemes provide

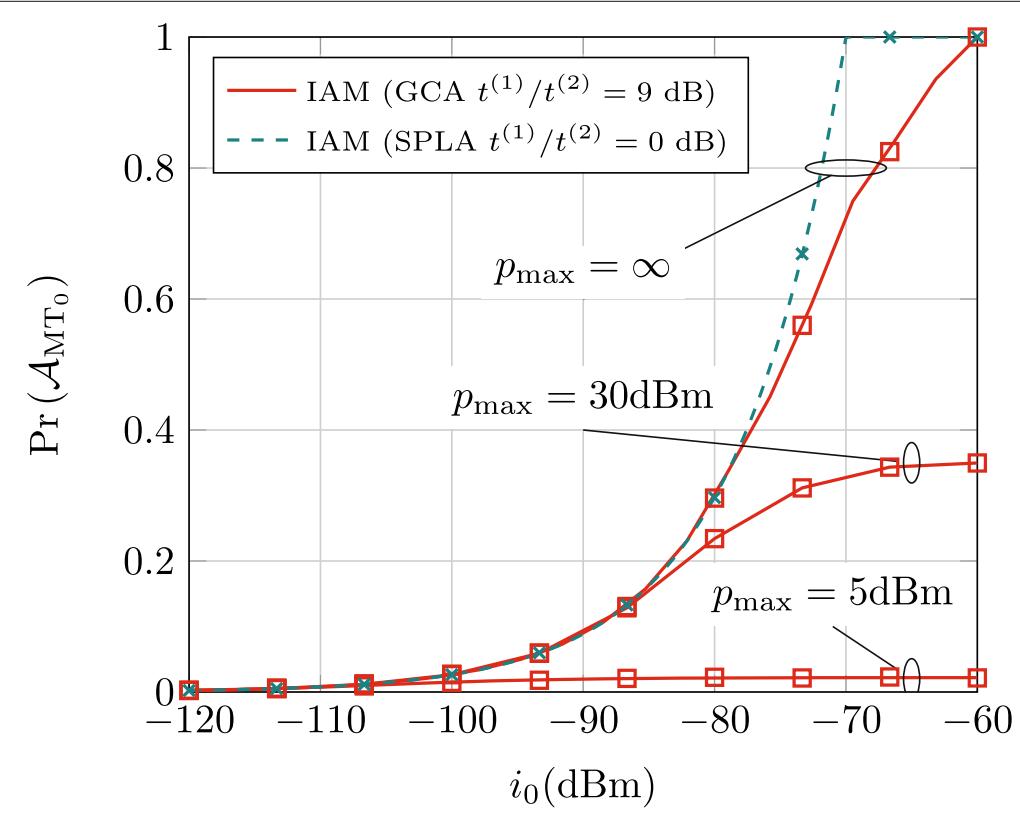

Fig. 2 Probability of being active of the typical MT for IAM with $\epsilon=1, p_{\max } \rightarrow \infty, p_{\max }=30 \mathrm{dBm}$, and $p_{\max }=5 \mathrm{dBm}$ 


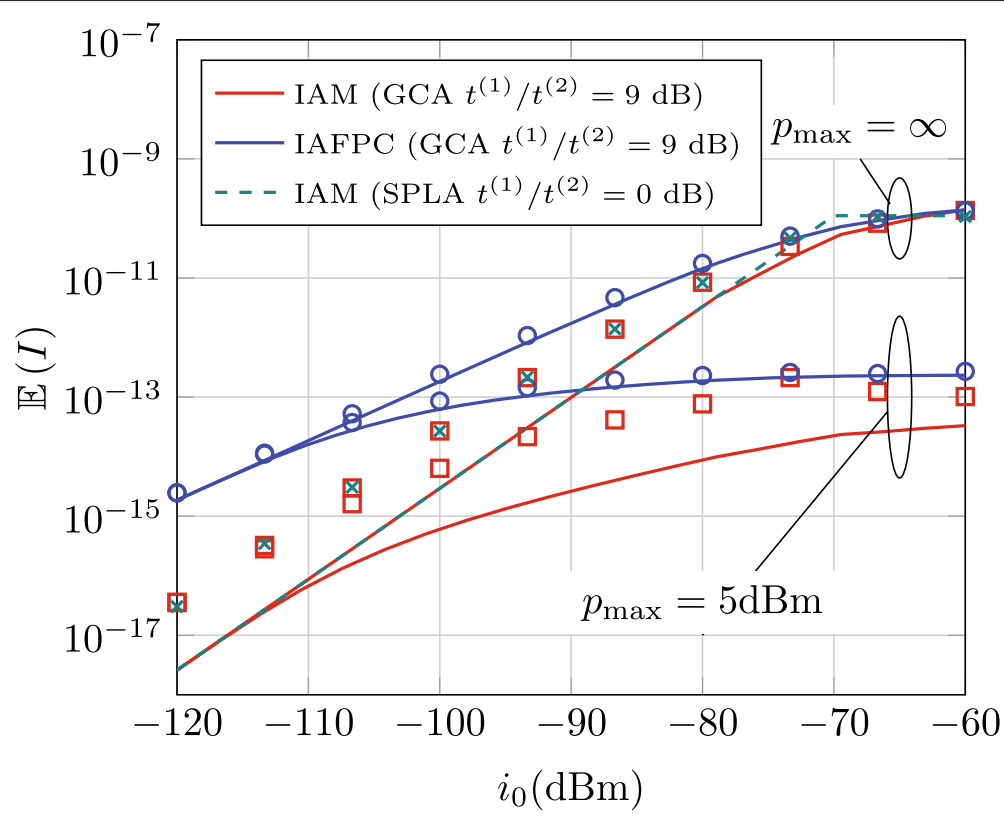

Fig. 3 Mean of the interference versus $i_{0}$ for IAM and IAFPC methods with $\epsilon=1, p_{\max } \rightarrow \infty$, and $p_{\max }=5 \mathrm{dBm}$

opposite trends compared to those evinced from the analysis of the average SE of the typical MT. More precisely, IAM provides a better average BR than IAFPC, and there exists an optimal value of $i_{0}$ that maximizes it. This optimal value of $i_{0}$ emerges if the typical MT is considered, i.e., the MT may be either active or inactive. The figure, however, shows the average BR achieved only by the active MTs. In this case, we note that the MTs that satisfy both power and interference constraints achieve a very high throughput due to the reduced level of interference that is generated in this case. In a nutshell, IAM outperforms IAFPC in terms of average BR because the available bandwidth is shared among fewer MTs (only those active), which results in a higher throughput for each of them. Even though some MTs may be turned off in IAM, this may not necessarily be considered as a downside from the

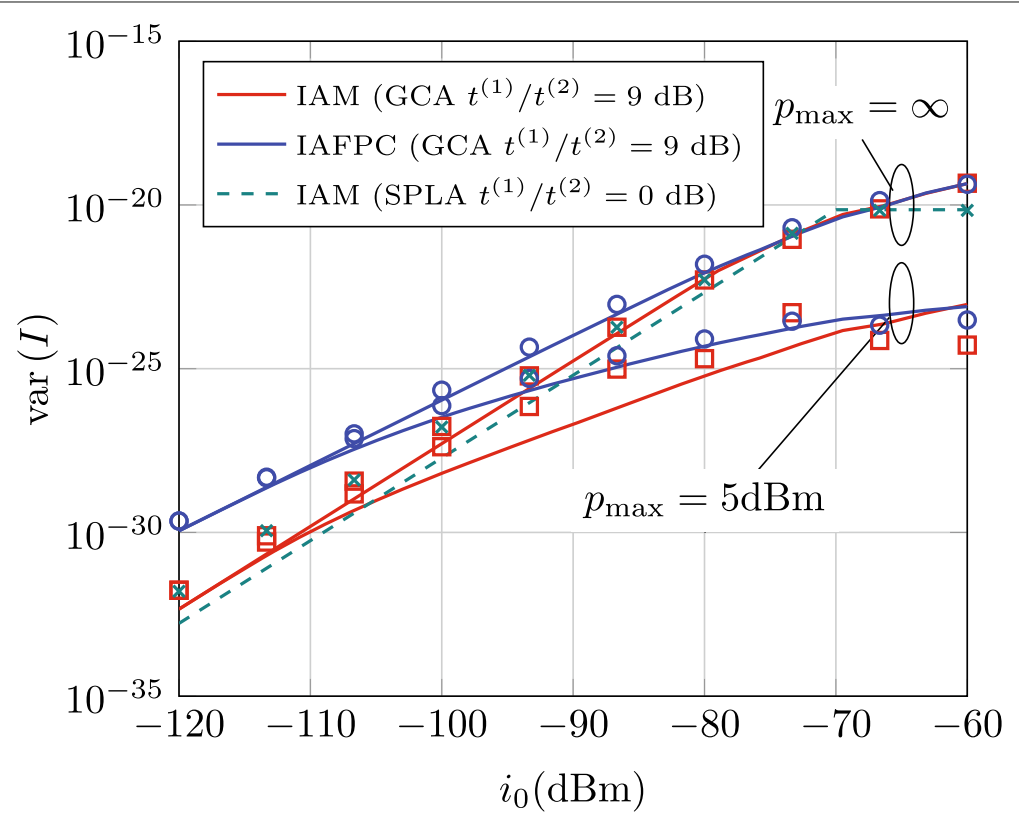

Fig. 4 Variance of the interference versus $i_{0}$ for IAM and IAFPC schemes with $\epsilon=1, p_{\max } \rightarrow \infty$, and $p_{\max }=5 \mathrm{dBm}$ 


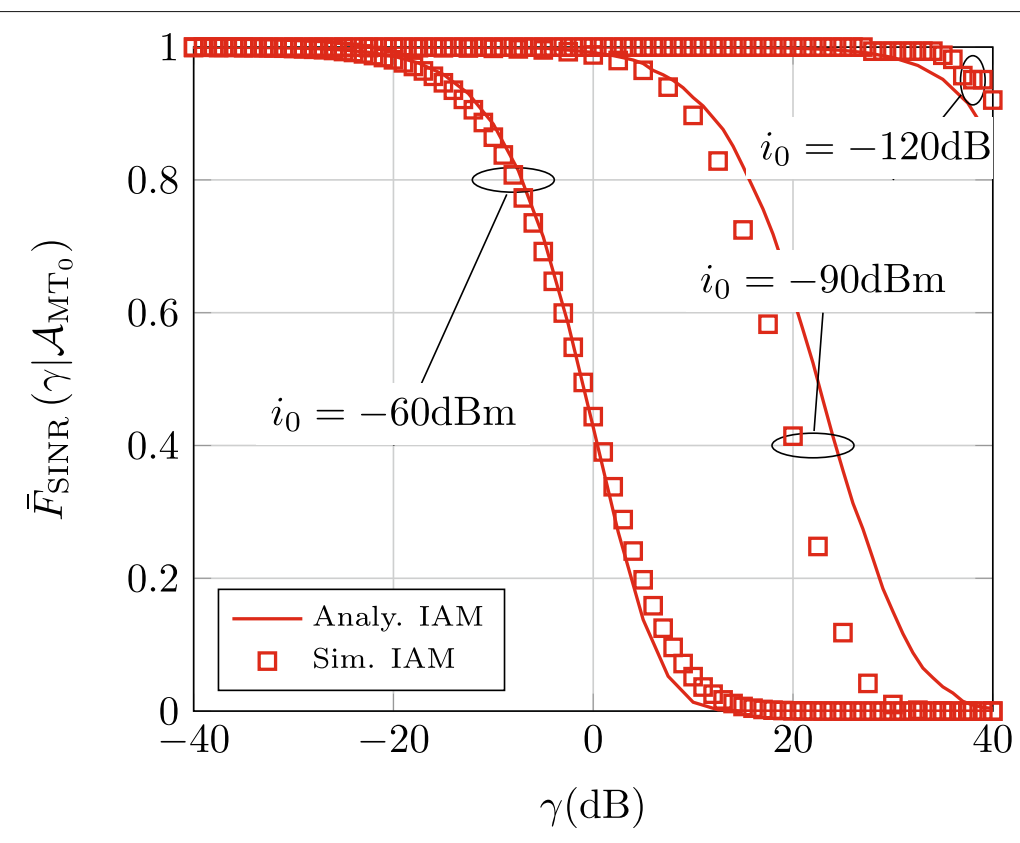

Fig. 5 CCDF of the SINR for the typical MT conditioned on being active for IAM with $\epsilon=1, t^{(1)} / t^{(2)}=9 \mathrm{~dB}, p_{\max } \rightarrow \infty$, and $i_{0}=\{-120,-90,-60\} \mathrm{dBm}$

user's perspective: in high-mobility scenarios, for example, some MTs may prefer to be muted for some periods of time if their reward is achieving a higher throughput once they are allowed to transmit. In Fig. 9, we study the impact of $p_{\max }$ for a given maximum interference constraint $i_{0}$. We observe that $p_{\max }$ plays a critical role as well and highly affects the average BR. This figure confirms, once again, that both $p_{\max }$ and $i_{0}$ constraints need to be appropriately optimized in order for IAM to outperform IAFPC.

In Fig. 10, we illustrate the potential of IAM of reducing the variance of the interference compared with IUM,

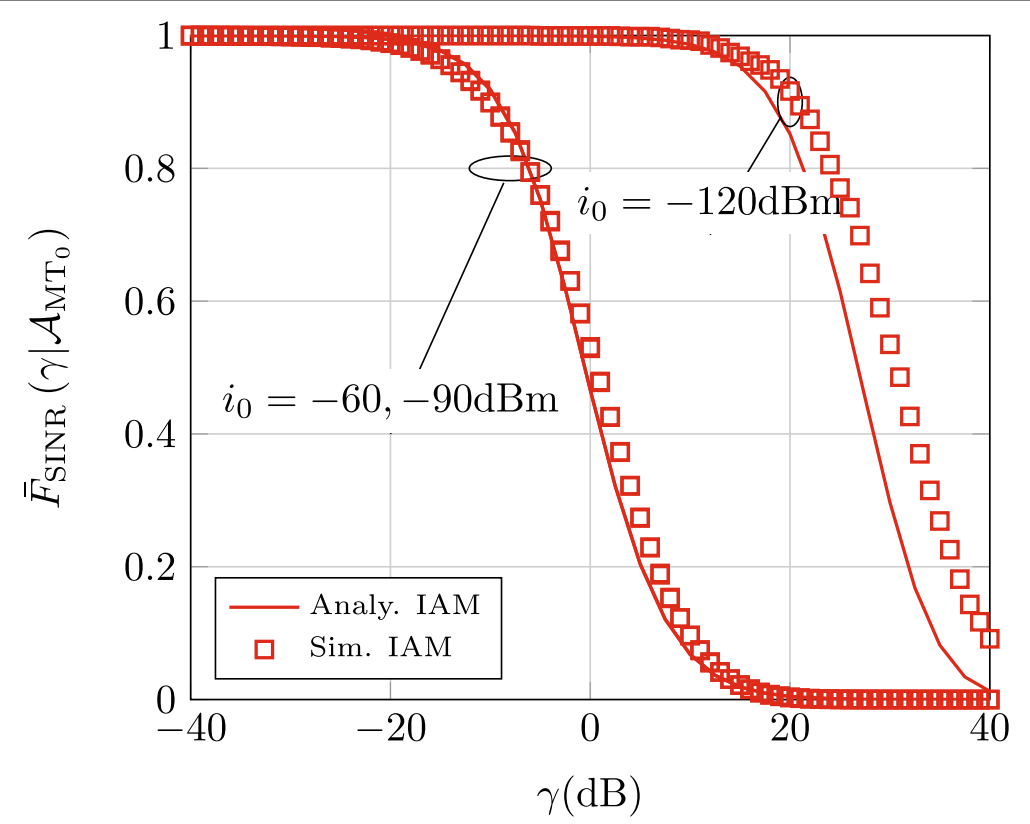

Fig. 6 CCDF of the SINR for the typical MT conditioned on being active for IAM with $\epsilon=0.75, t^{(1)} / t^{(2)}=9 \mathrm{~dB}, p_{\max } \rightarrow \infty$ and $i_{0}=\{-120,-90,-60\} \mathrm{dBm}$ 


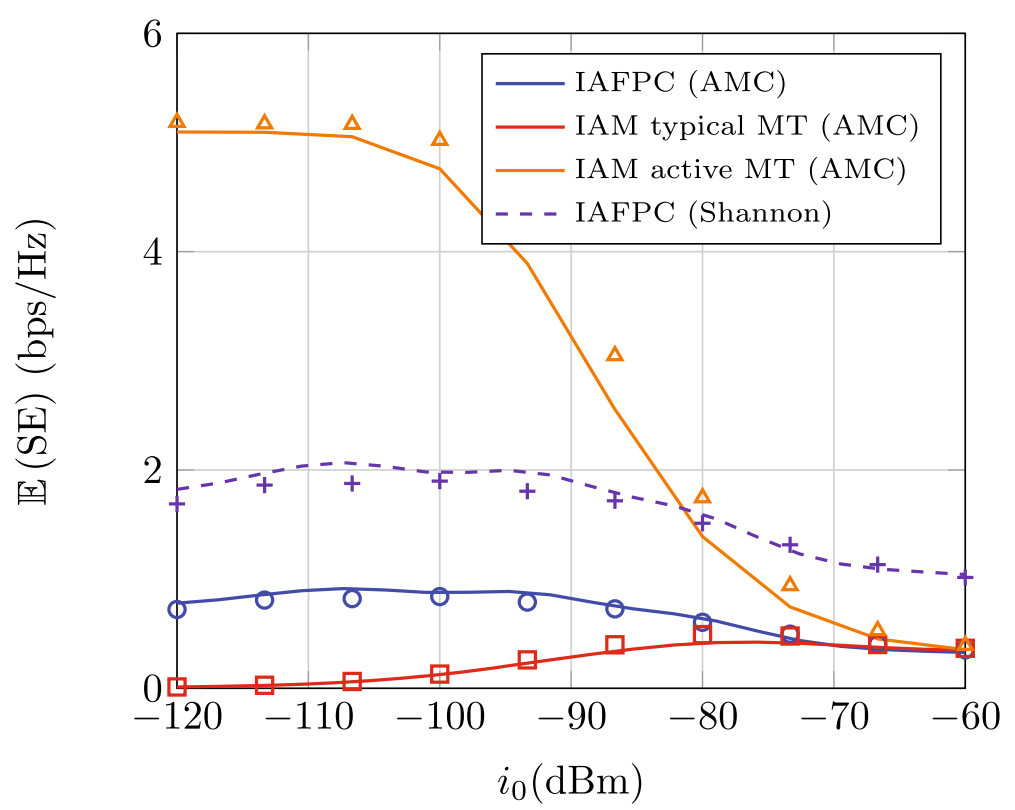

Fig. 7 Comparison of average SE of IAFPC and IAM for $\epsilon=1, t^{(1)} / t^{(2)}=9 \mathrm{~dB}$ and $p_{\max } \rightarrow \infty$. As for IAFPC, the average SE based on the Shannon formula is shown as well

while still guaranteeing the same average BR. As discussed in the previous sections, this is beneficial for implementing AMC schemes. The figure shows a four-order magnitude reduction of the variance of the interference for the considered setup of parameters.

\subsection{Impact of the association weights: on UL-DL decoupling}

As shown in [2] and [5], optimizing the performance of HCNs for DL transmission does not necessarily results in optimizing their performance in the UL. Based on

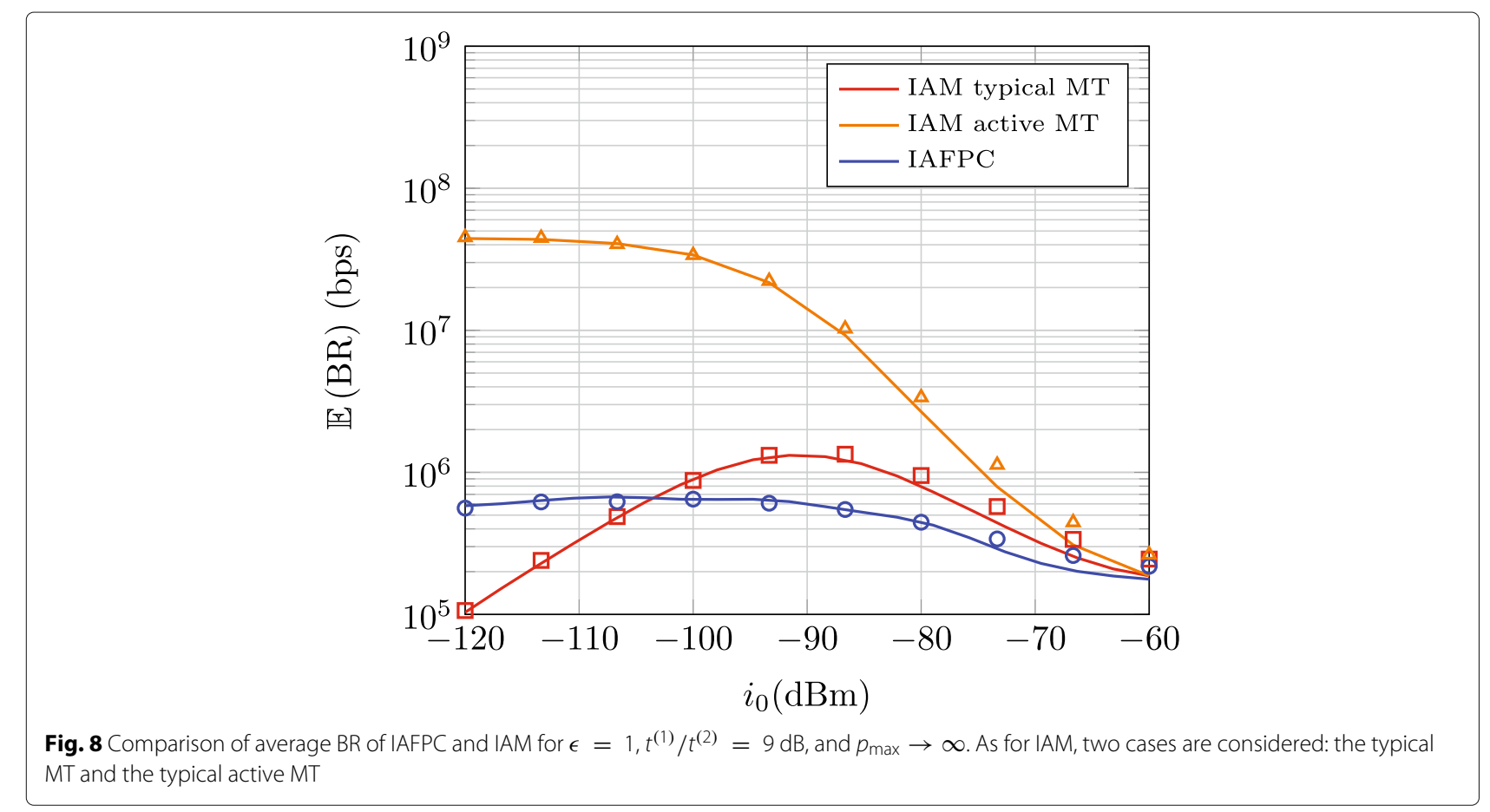




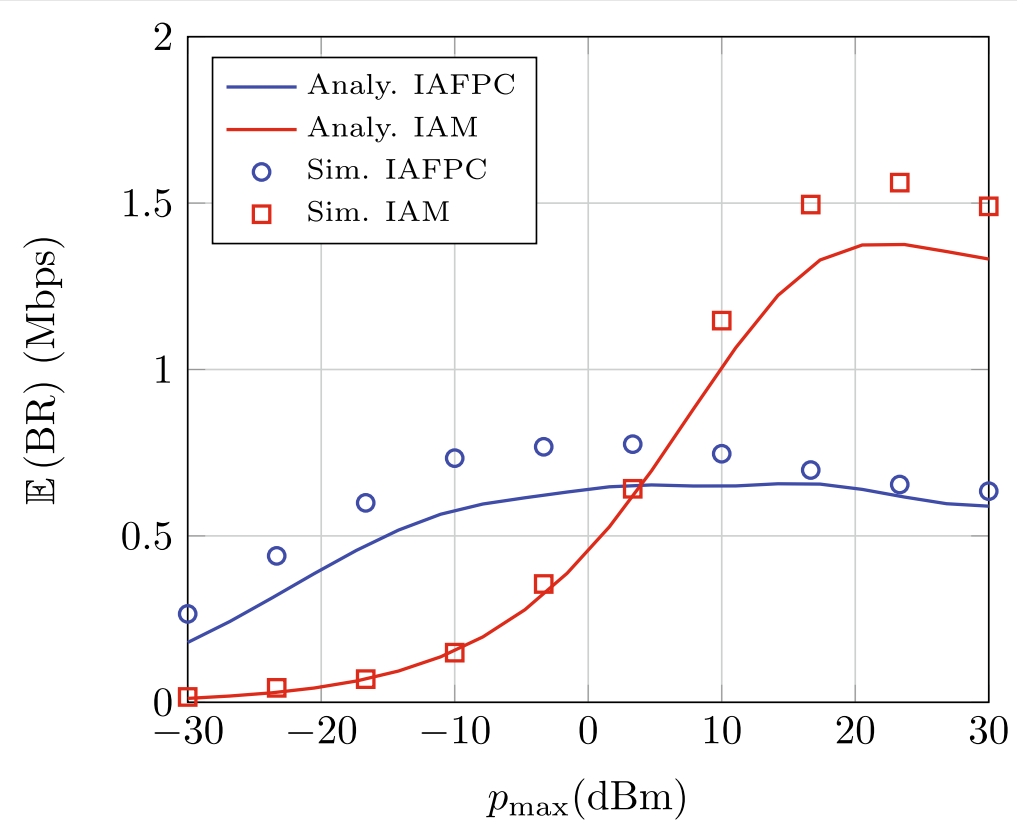

Fig. 9 Comparison of average BR of IAFPC and IAM for $\epsilon=1, t^{(1)} / t^{(2)}=9 \mathrm{~dB}$ and $i_{0}=-90 \mathrm{dBm}$

the GCA criterion, this implies that different cell association weights (i.e., a different ratio $t^{(1)} / t^{(2)}$ for two-tier $\mathrm{HCNs}$ ) may be needed in the DL and in the UL. However, this approach, which is referred to as UL-DL decoupling, introduces additional implementation challenges, which require the modification of the existing network architecture and control plane.
In this section, motivated by these considerations, we analyze and compare IAM, IAFPC, and IUFPC schemes as a function of $t^{(1)} / t^{(2)}$. The setup $t^{(1)} / t^{(2)}=0 \mathrm{~dB}$ corresponds to the SPLA criterion. Some numerical illustrations are provided in Figs. 11 and 12, where the probability that the typical MT is active and the average BR are shown, respectively.

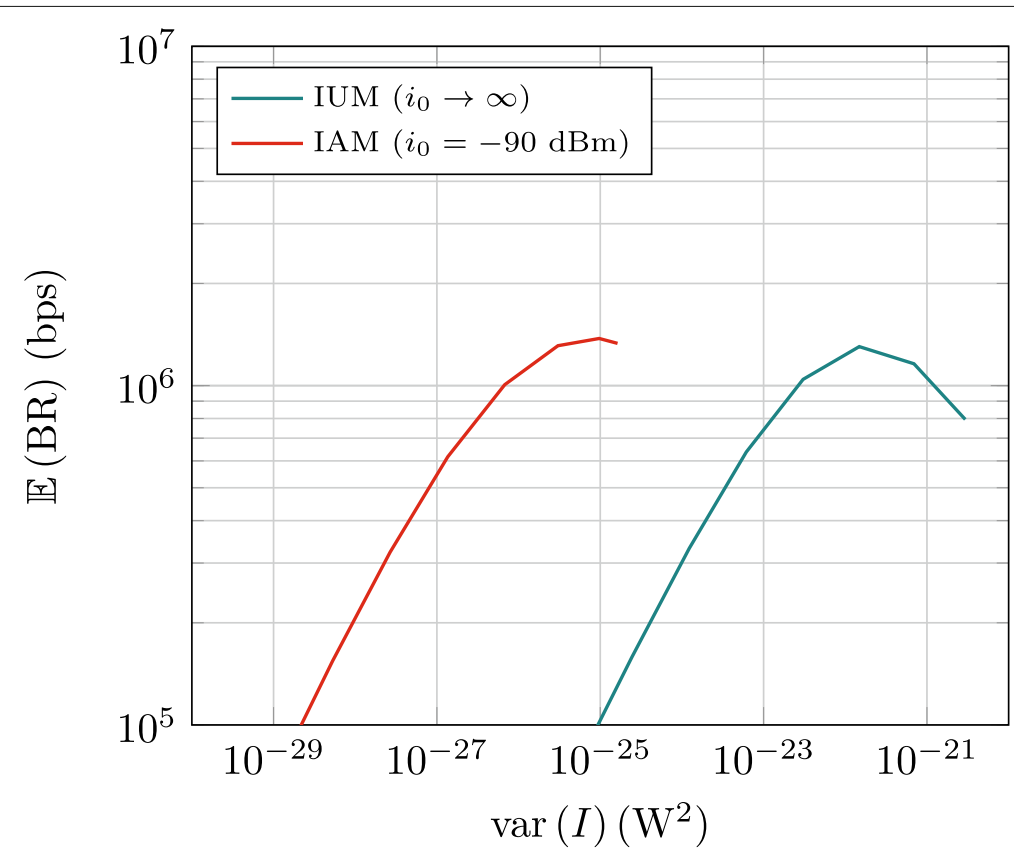

Fig. 10 Average BR as a function of the variance of the interference for IAM $\left(i_{0}=-90 \mathrm{dBm}\right)$ and IUM $\left(i_{0} \rightarrow \infty\right)$ schemes with $p_{\max }=5 \mathrm{dBm}$ and $t^{(1)} / t^{(2)}=9 \mathrm{~dB}$ 


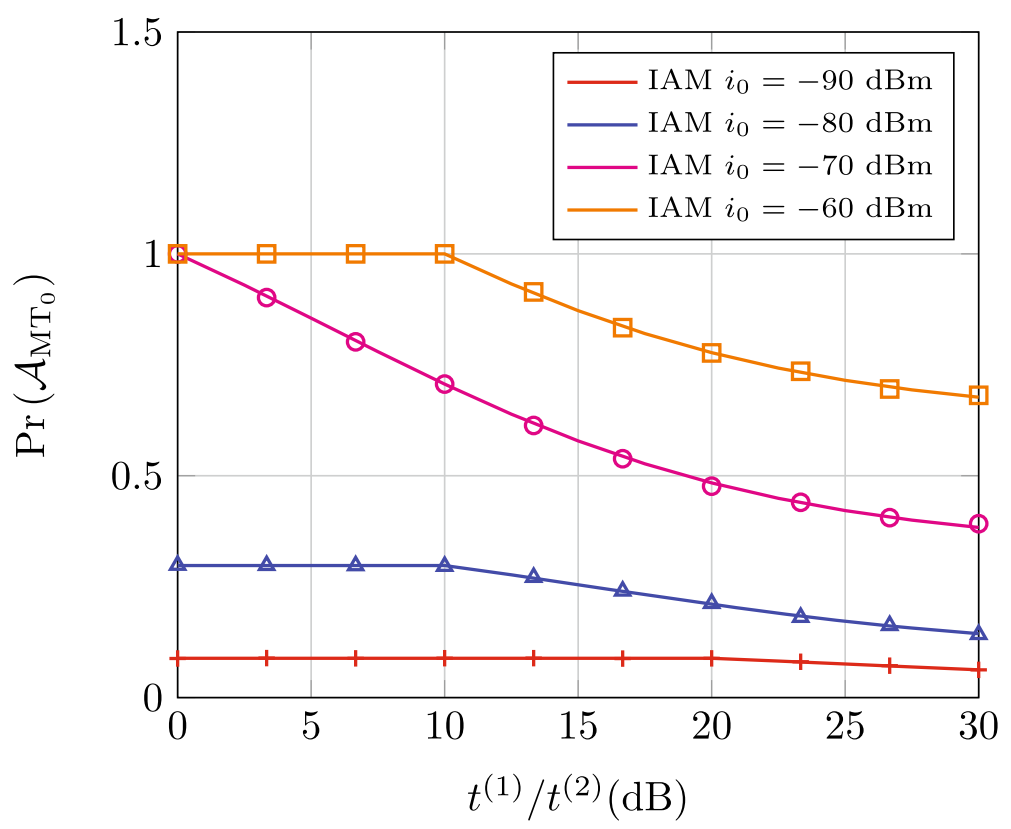

Fig. 11 Probability the typical MT is active as a function of $t^{(1)} / t^{(2)}$ for IUFPC $\left(i_{0} \rightarrow \infty\right)$ and IAM with $i_{0}=\{-90,-80,-70,-60\}$ BBm. $p_{\max } \rightarrow \infty$ for both schemes

In Fig. 12, in particular, we compare the average BR of IUFPC and IAM schemes. The figure highlights important differences between these two interference management schemes for improving the performance of the UL of HCNs. First of all, we note that the average BR of the IUFPC scheme decreases as the ratio $t^{(1)} / t^{(2)}$ increases. More specifically, the best average $\mathrm{BR}$ is obtained if the SPLA criterion is used, which is in agreement with previously published papers [16]. This originates from the fact that the larger $t^{(1)} / t^{(2)}$ is, the more MTs are associated with more distance BSs, which, due to the use of power control, results in increasing the interference in the UL. The performance trend is, on the other hand, different if the IAM scheme is used. In this case, there are

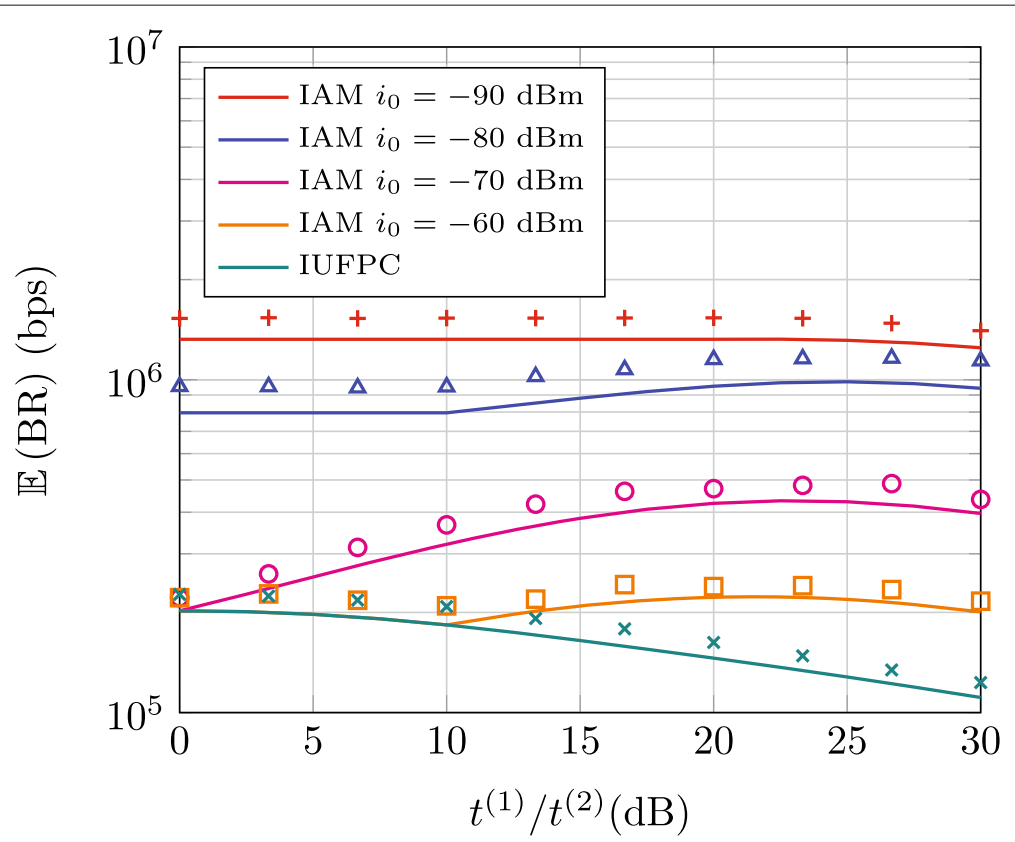

Fig. 12 Average BR as a function of $t^{(1)} / t^{(2)}$ for IUFPC $\left(i_{0} \rightarrow \infty\right)$ and IAM with $i_{0}=\{-90,-80,-70,-60\} \mathrm{dBm}$. $p_{\max } \rightarrow \infty$ for both schemes 
several values of $i_{0}$ that provide a better average BR compared with IUFPC. In addition, the average BR increases as $t^{(1)} / t^{(2)}$ increases, since the excess interference that is generated under the IUFPC scheme is now kept under control by imposing the maximum interference constraint $i_{0}$. As observed in previous figures, Fig. 11 confirms that this gain is obtained since more MTs are turned off.

Figures 11 and 12 confirm the findings in Remark 3 and, in particular, the existence of an operating regime where the performance of IAM is independent of the association weights. Let us consider, for example, the setup for $i_{0}=-60 \mathrm{dBm}$. In this case, $i_{0}>p_{0}$ and hence, according to Remark 3, the system is interference-unaware if $t^{(1)} / t^{(2)} \in[-10,+10] \mathrm{dB}$. Figure 12 , more specifically, confirms that IAM is interference-unaware since it provides the same average BR as IUFPC for $t^{(1)} / t^{(2)} \in$ $[-10,+10] \mathrm{dB}^{6}$. Similar conclusions can be drawn for other values of $i_{0}$, where different operating regimes can be identified as predicted in Remark 3. If $i_{0}=-90 \mathrm{dBm}$, in particular, then $i_{0}<p_{0}$ and the system is independent of the cell association criterion for $t^{(1)} / t^{(2)} \in[-20,+20]$, which is confirmed in Figs. 11 and 12. It is worth mentioning that the values of $t^{(1)} / t^{(2)}$ for which the considered system model is cell association independent are usually adopted in practical engineering applications. In particular, the authors of $[2,19]$ have shown that the optimal cell association ratio that optimizes the DL is usually less than $20 \mathrm{~dB}$. This is in agreement and compatible with the findings in Figs. 11 and 12.

In view of the numerical results and theoretical insights derived in this work, it is possible to state the following arguments in favor of IAM:

1 Taking into account the periods where the typical MT is active and those where it is muted, the average $\mathrm{BR}$ is increased with IAM compared to IAFPC and IUFPC.

2 Thanks to mobility and shadowing, MTs are only muted for a given period of time.

3 Since the muted MTs do not transmit, their average transmitted power is reduced compared to IAFPC and IUFPC. This has been studied with Fig. 1.

4. With IAM, there is a regime where the UL performance is independent of cell association, which eases the joint design of UL and DL transmissions as it have been discussed above.

5 The IAM scheme can be further enhanced as discussed in Section 7. Some numerical illustrations are provided in the next section.

\subsection{Hybrid scheme-complementary cumulative distribution function of the SINR}

In this section, we analyze the coverage probability (CCDF of the SINR) of the hybrid scheme introduced in Section 7.
The results are illustrated in Figs. 13, 14, and 15 for $i_{0}=-70, i_{0}=-90$, and $i_{0}=-120$, respectively, and by assuming $\epsilon=1$ and $p_{\max } \rightarrow \infty$.

In these figures, we observe a good agreement between analytical frameworks and Monte Carlo simulations.

In Fig. 13, it can be observed that the coverage probability of the hybrid scheme increases as $t_{1}$ increases. In Fig. 14, it can be observed that the coverage probability of the hybrid scheme decreases first and then increases as $t_{1}$ increases. In Fig. 15, it can be observed that coverage probability of the hybrid scheme decreases as $t_{1}$ decreases. As for high $i_{0}$, these trends are obtained because the coverage probability in time slot 1 is better than in time slot 2 for high $i_{0}$ since more MTs are active. Then, increasing $t_{1}$ improves the coverage probability. As for low $i_{0}$, the trend is opposite for similar reasons. The comparison of these figures also shows that the coverage probability of the hybrid scheme decreases as $i_{0}$ increases for small value of $t_{1}$ because time slot 2 dominates the performance. On the contrary, the coverage probability of the hybrid scheme increases as $i_{0}$ increases for large value of $t_{1}$.

\subsection{Hybrid scheme-spectral efficiency and binary rate}

In this section, the average $\mathrm{SE}$ and average $\mathrm{BR}$ are analyzed.

In Fig. 16, the average $\mathrm{SE}$ of the hybrid system is reported. By comparing the average SE of the typical MTs, we note a different performance trend as a function of $i_{0}$ for different values of $t_{1}$. If $t_{1}=1$, which is the IAM scheme, the average SE increases as $i_{0}$ increases as discussed in the previous sections. If $t_{1}=0$, only the muted MTs transmit signals and the average SE decreases as $i_{0}$ increases. If, e.g., $t_{1}=0.5$, the average SE increases first and then decreases as $i_{0}$ increases.

This result is reasonable because the lower $i_{0}$ is the fewer MTs transmit in time slot 1 and more MTs transmit in time slot 2, which on average, contributes to reduce the $\mathrm{SE}$ if $t_{1}=1$ and to increase the SE if $t_{1}=0$. As for the hybrid scheme, the $\mathrm{SE}$ is somehow in between.

The average BR for different values of $t_{1}$ is illustrated in Fig. 17. We note that the average BRs are quite similar if $t_{1}=0$, while the gap increases if $t_{1}=1$. We observe a quite large gap as a function of $t_{1}$. This highlights that $t_{1}$ should be carefully allocated in order to fulfill system fairness and interference mitigation. The proposed mathematical frameworks can be used to optimize these competing performance metrics.

\section{Conclusion}

In this paper, we have studied the performance of IAM: an interference management scheme for enhancing the 


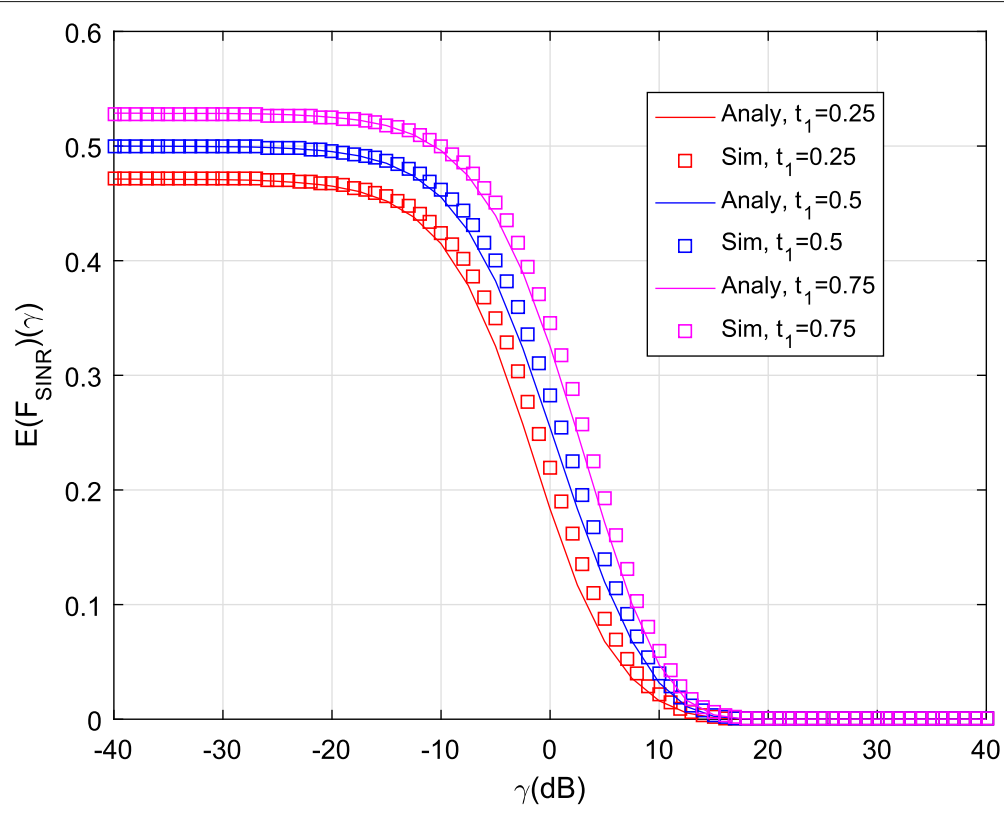

Fig. 13 CCDF of the SINR of the hybrid scheme for the typical MT with $\epsilon=1, t^{(1)} / t^{(2)}=9 \mathrm{~dB}, p_{\max } \rightarrow \infty$ and $i_{0}=-70 \mathrm{dBm}$

throughput of HCNs. With the aid of stochastic geometry, we have developed a general mathematical approach for analyzing and optimizing its performance as a function of several system parameters. Simplified and insightful expressions of the throughput and other relevant performance indicators have been proposed for simplified but relevant case studies, such as in the presence of channel inversion power control and equal cell association weights. Among the many performance trends that have been identified, we have proved that, while optimizing the DL and the UL of HCNs necessitates, in general, to use different cell association weights, there exist some operating regimes where IAM is cell association independent. This is shown to simplify the design

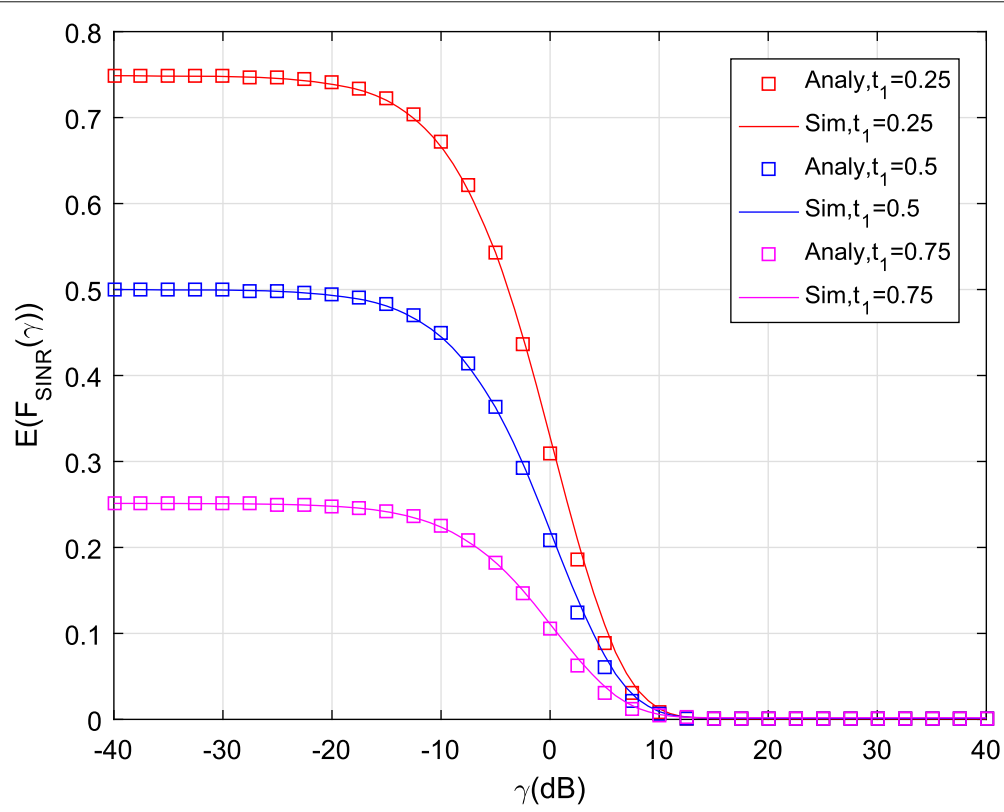

Fig. 14 CCDF of the SINR of the hybrid scheme for the typical MTM with $\epsilon=1, t^{(1)} / t^{(2)}=9 \mathrm{~dB}, p_{\max } \rightarrow \infty$, and $i_{0}=-90 \mathrm{dBm}$ 


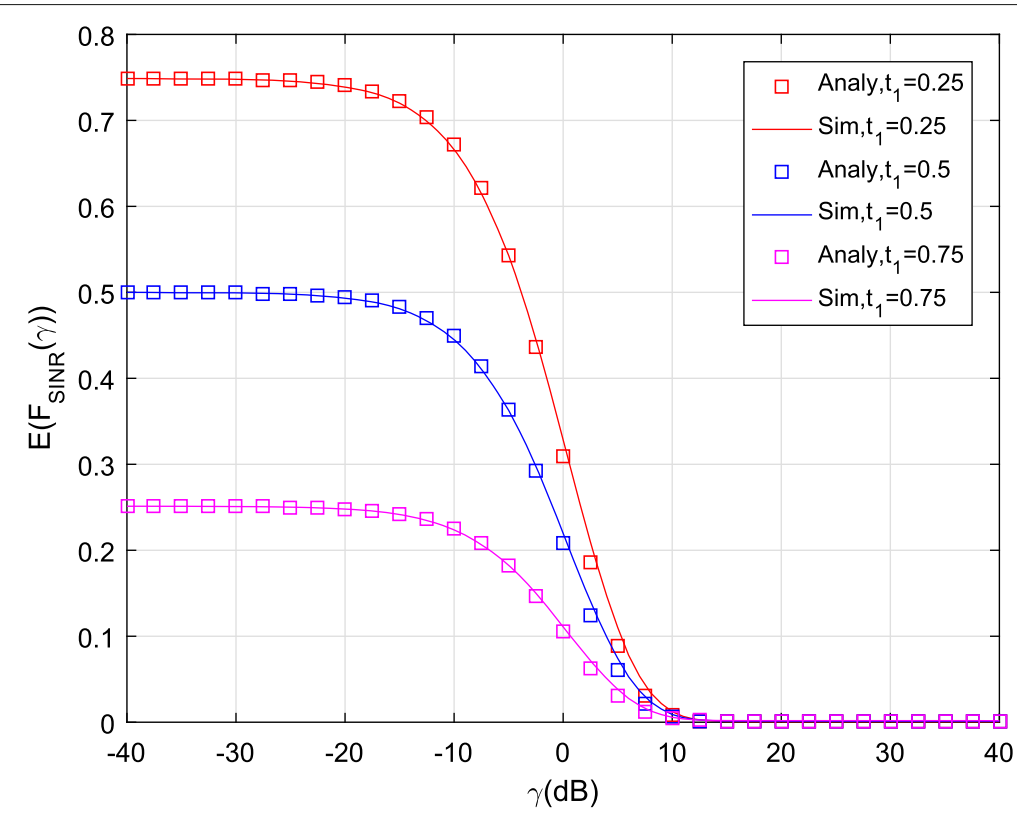

Fig. 15 CCDF of the SINR of the hybrid scheme for the typical MT with $\epsilon=1, t^{(1)} / t^{(2)}=9 \mathrm{~dB}, p_{\max } \rightarrow \infty$, and $i_{0}=-120 \mathrm{dBm}$

of HCNs, since no changes in their control plane is needed compared with conventional cellular networks. The mathematical frameworks and findings have been substantiated against Monte Carlo simulations, as well as the achievable performance of IAM has been compared against other IAFPC and IUFPC schemes, by highlighting several important trade-offs in terms of system fairness and system throughput. Finally, we have introduced a hybrid scheme that overcomes some fairness issues associated with the operating principle of the IAM scheme, and have highlighted important performance trade-offs.

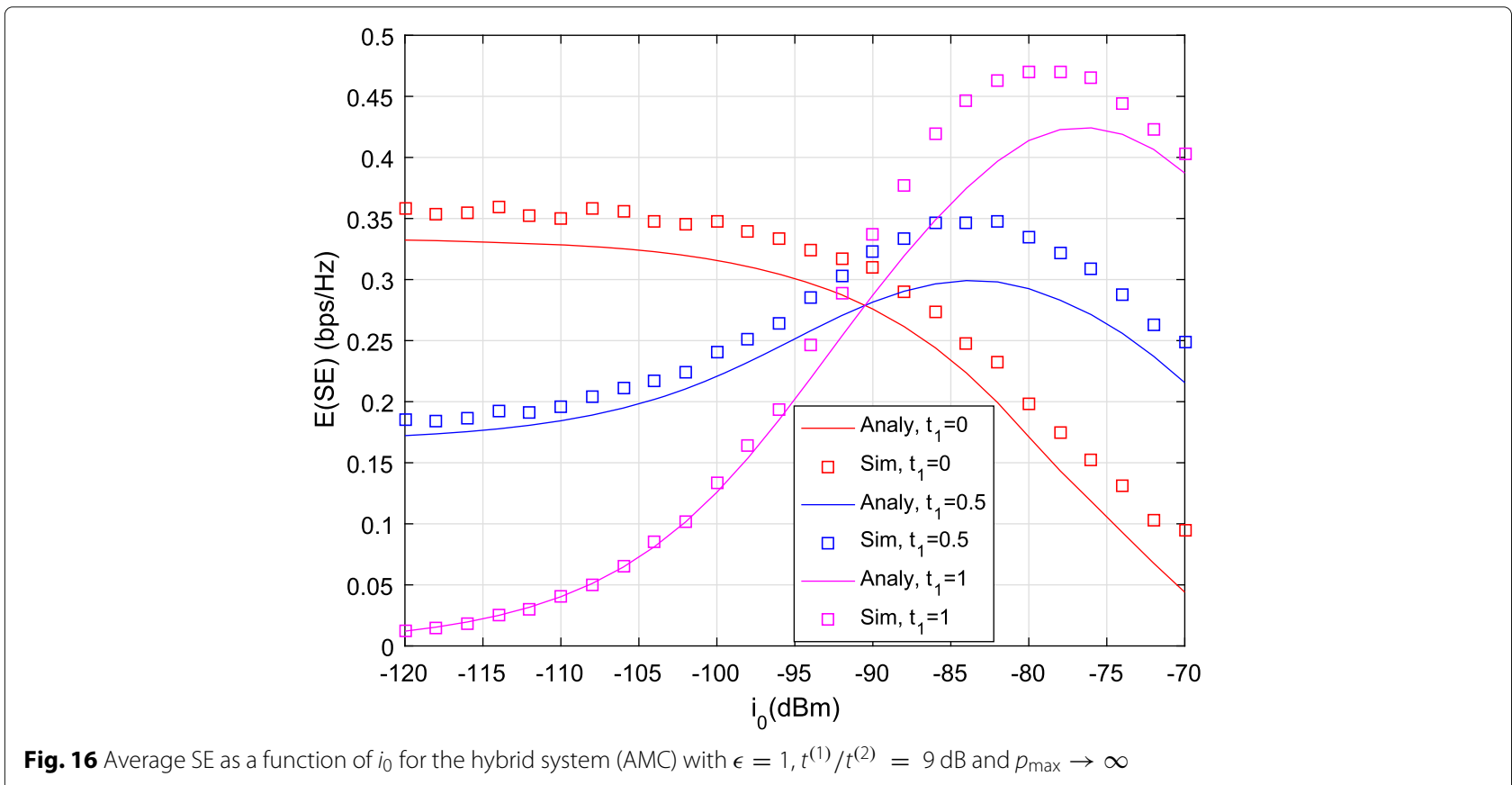

Fig. 16 Average $S E$ as a function of $i_{0}$ for the hybrid system (AMC) with $\epsilon=1, t^{(1)} / t^{(2)}=9 \mathrm{~dB}$ and $p_{\max } \rightarrow \infty$ 


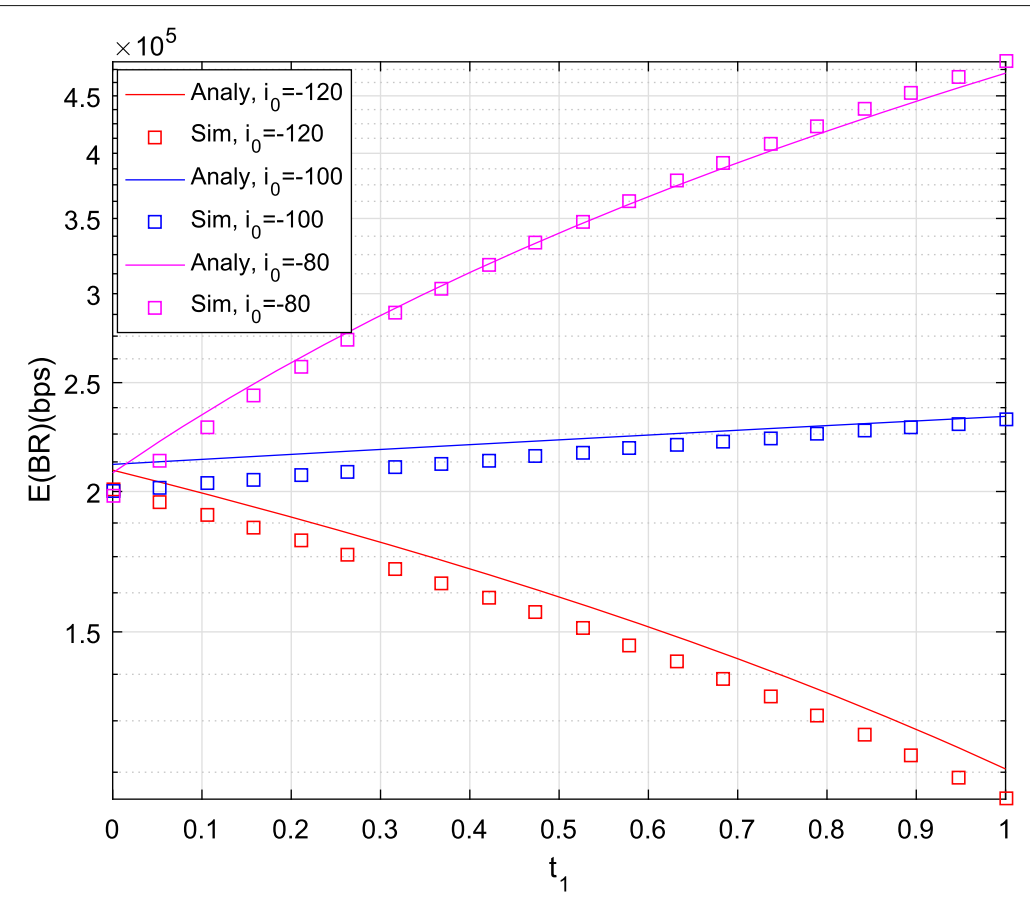

Fig. 17 Average $B R$ as a function of the transmitting time in time slot 1 for the hybrid system (AMC) with $\epsilon=1, t^{(1)} / t^{(2)}=9 \mathrm{~dB}$ and $p_{\max } \rightarrow \infty$

\section{Endnotes}

${ }^{1}$ The proposed framework can be generalized to account for a bounded path loss model; however, an unbounded path loss model has been used for the sake of mathematical tractability.

${ }^{2}$ Throughout this paper, all the distances implicitly include shadowing.

${ }^{3}$ Although, in practice, the bandwidth is divided in RBs, we assume that it can be treated as a continuous resource and hence that it can be equally divided among the active MTs. This is assumed in [2] as well.

${ }^{4}$ In the present paper, IUM and interference unaware FPC (IUFPC) schemes are similar but slightly different. IUM is referred to a setup where $i_{0} \rightarrow \infty$ and $p_{\max }<$ $\infty$. IUFPC is referred to a setup where $i_{0} \rightarrow \infty$ and $p_{\max } \rightarrow \infty$. As for IUM, only the constraint on the maximum transmit power exists. As for IUFPC, there is no constraint on either the maximum transmit power or the maximum interference.

${ }^{5}$ The system fairness can be defined in different ways. In [20], e.g., it is defined based on the proportionally fair criterion and is obtained by computing the logarithm of the average rate. Our framework could be generalized for analyzing the system fairness based on this definition.

${ }^{6}$ Only positive values (in decibels) of the association weights $t^{(1)} / t^{(2)}$ are shown in Figs. 11 and 12.

\section{Appendix}

\section{A. Proof of Proposition 1}

The probability that a MT is active is by definition as follows:

$$
\operatorname{Pr}\left(\mathcal{A}_{\mathrm{MT}_{0}}\right)=\sum_{j \in \mathcal{K}} \sum_{m \in \mathcal{K}} \operatorname{Pr}\left(\mathcal{X}_{\mathrm{MT}_{0}}^{(j, m)}, \mathcal{A}_{\mathrm{MT}_{0}}\right)
$$

where $\operatorname{Pr}\left(\mathcal{X}_{\mathrm{MT}_{0}}^{(j, m)}, \mathcal{A}_{\mathrm{MT}_{0}}\right)$ is the probability that the MT is active, is associated to tier $j$ and that the most interfered BS belongs to tier $m$. If $j \neq m$, it can be written as follows:

$$
\begin{gathered}
\operatorname{Pr}\left(\mathcal{X}_{\mathrm{MT}_{0}}^{(j, m)}, \mathcal{A}_{\mathrm{MT}_{0}}\right) \stackrel{(\mathrm{a})}{=} \mathbb{E}_{R_{\mathrm{MT}_{0},(1)}^{(m)}} \mathbb{E}_{R_{\mathrm{MT}_{0},(1)}^{(j)}, R_{\mathrm{MT}_{0},(2)}^{(j)}} \\
\quad \mathbf{1}\left(R_{\mathrm{MT}_{0},(1)}^{(m)}>\left(\frac{t^{(m)}}{t^{(j)}}\right)^{\frac{1}{\alpha}} R_{\mathrm{MT}_{0},(1)}^{(j)}\right) \times \\
\mathbf{1}\left(R_{\mathrm{MT}_{0},(1)}^{(m)}<R_{\mathrm{MT}_{0},(2)}^{(j)}\right) \times \\
\mathbf{1}\left(R_{\mathrm{MT}_{0},(1)}^{(j)}<\frac{1}{\tau}\left(\frac{p_{\max }}{p_{0}}\right)^{\frac{1}{\alpha \epsilon}}\right) \times \\
\left.\mathbf{1}\left(R_{\mathrm{MT}_{0},(1)}^{(m)}>\left(\frac{p_{0}}{i_{0}}\right)^{\frac{1}{\alpha}} \frac{\left(\tau R_{\mathrm{MT}_{0},(1)}^{(j)}\right)}{\tau}\right)\right]
\end{gathered}
$$

where (a) is obtained by definition of expectation formulated with the aid of indicator functions. 
To compute this expectation, the PDF of the distance of the nearest BS and of the joint PDF of the distances of the nearest and second nearest BSs are needed. By definition of PPP, they are equal to $f_{R_{\mathrm{MT}_{0},(1)}^{(j)}}(r)=2 \pi \lambda^{(j)} r \mathrm{e}^{-\pi \lambda^{(j)} r^{2}}$ and

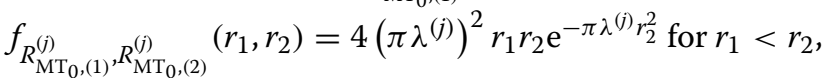
respectively, [21]. With the aid of these PDFs, we obtain:

$$
\begin{aligned}
& \operatorname{Pr}\left(\mathcal{X}_{\mathrm{MT}_{0}}^{(j, m)}, \mathcal{A}_{\mathrm{MT}_{0}}\right)=\int_{r_{1}^{(j)}=0}^{\frac{1}{\tau}\left(\frac{p_{\max }}{p_{0}}\right)^{\frac{1}{\alpha \epsilon}}} \\
& \int^{\infty} \quad f_{R_{\mathrm{MT}_{0},(1)}^{(m)}}\left(r_{1}^{(m)}\right) \\
& r_{1}^{(m)}=\max \left(\left(\frac{p_{0}}{i_{0}}\right)^{\frac{1}{\alpha}} \frac{\left(\tau r_{1}^{(j)}\right)^{\epsilon}}{\tau},\left(\frac{t^{(m)}}{t^{(j)}}\right)^{\frac{1}{\alpha}} r_{1}^{(j)}\right) \\
& \int_{(j)(m)}^{\infty} f_{R_{\mathrm{MT}_{0},(1)}^{(j)}, R_{\mathrm{MT}_{0},(2)}^{(j)}}\left(r_{1}^{(j)}, r_{2}^{(j)}\right) \mathrm{d} r_{2}^{(j)} \mathrm{d} r_{1}^{(m)} \mathrm{d} r_{1}^{(j)} \\
& r_{2}^{(j)}=\max \left(r_{1}^{(j)}, r_{1}^{(m)}\right) \\
& \varphi\left(r_{1}^{(j)}, r_{1}^{(m)}\right)
\end{aligned}
$$

The computation of the two-fold integral leads to the function $v^{(j)}(v)$ that is provided in (9).

The case $j=m$ can be solved by using an approach similar to the previous case. The final result corresponds to the function $\eta^{(j)}(v)$ available in (10).

By combining both cases $j=m$ and $j \neq m$, $\operatorname{Pr}\left(\mathcal{X}_{\mathrm{MT}_{0}}^{(j, m)}, \mathcal{A}_{\mathrm{MT}_{0}}\right)$ can be written as follows:

$$
\begin{aligned}
& \operatorname{Pr}\left(\mathcal{X}_{\mathrm{MT}_{0}}^{(j, m)}, \mathcal{A}_{\mathrm{MT}_{0}}\right)=\int_{0}^{\frac{1}{\tau}\left(\frac{p_{\max }}{p_{0}}\right)^{\frac{1}{\alpha \epsilon}}} \\
& \quad\left(\mathbf{1}(j \neq m) v^{(j, m)}(v)+\mathbf{1}(j=m) \eta^{(j)}(v)\right) \mathrm{d} v
\end{aligned}
$$

The proof follows by computing the summation over $m \in \mathcal{K}$ in (58).

\section{B. Proof of Lemma 2}

The Laplace transform of the interference can be expressed as follows:

$$
\begin{gathered}
\mathcal{L}_{I}\left(s \mid \mathcal{X}_{\mathrm{MT}_{0}}^{(j)}\right)=\mathbb{E}_{I}\left[\mathrm{e}^{-s I} \mid \mathcal{X}_{\mathrm{MT}_{0}}^{(j)}\right]=\prod_{k \in \mathcal{K}} \mathbb{E}_{\Psi^{(k)}} \\
\prod_{\mathrm{MT}_{i} \in \Psi^{(k)}} \mathbb{E}_{R_{\mathrm{MT}_{i}}}\left[\mathbb { E } _ { H _ { \mathrm { MT } _ { i } } } \operatorname { e x p } \left(-s H_{\mathrm{MT}_{i}}\left(\tau D_{\mathrm{MT}_{i}}\right)^{-\alpha}\right.\right. \\
\left.\left.\left(\tau R_{\mathrm{MT}_{i}}\right)^{\alpha \epsilon} p_{0} \mathbf{1}\left(\mathcal{O}_{\mathrm{MT}_{i}}^{(j, k)}\right) \mathbf{1}\left(\mathcal{Z}_{\mathrm{MT}_{i}}\right)\right) \mid \mathcal{X}_{\mathrm{MT}_{i}}^{(k)}, \mathcal{A}_{\mathrm{MT}_{i}}\right]
\end{gathered}
$$

By applying the Probability Generating Functional (PGF) theorem in [11] and computing the expectation with respect to the channel fading, $\mathcal{L}_{I}\left(s \mid \mathcal{X}_{\mathrm{MT}_{0}}^{(j)}\right)$ is as follows:

$$
\begin{aligned}
& \exp \left(-\sum_{k \in \mathcal{K}} 2 \pi \lambda^{(k)} \int_{\rho=0}^{\infty} \mathbb{E}_{R_{\mathrm{MT}_{i}}}\left[\mathbf{1}\left(\mathcal{O}_{\mathrm{MT}_{i}}^{(j, k)}\right) \mathbf{1}\left(\mathcal{Z}_{\mathrm{MT}_{i}}\right)\right.\right. \\
& \left.\left.\frac{s(\tau \rho)^{-\alpha}\left(\tau R_{\mathrm{MT}_{i}}\right)^{\alpha \epsilon} p_{0}}{1+s(\tau \rho)^{-\alpha}\left(\tau R_{\mathrm{MT}_{i}}\right)^{\alpha \epsilon} p_{0}} \rho \mid \mathcal{X}_{\mathrm{MT}_{i}}^{(k)}, \mathcal{A}_{\mathrm{MT}_{i}}\right] \mathrm{~d} \rho\right)
\end{aligned}
$$

By conditioning on the event $\mathcal{Q}_{\mathrm{MT} i}^{(n)}$ defined in (2) and by using the total probability theorem, $\mathcal{L}_{I}\left(s \mid \mathcal{X}_{\mathrm{MT}_{0}}^{(j)}\right)$ can be written as follows:

$$
\begin{aligned}
& \exp \left(-\sum_{k \in \mathcal{K}} 2 \pi \lambda^{(k)} \sum_{n \in \mathcal{K}} \operatorname{Pr}\left(\mathcal{Q}_{\mathrm{MT}_{i}}^{(n)} \mid \mathcal{X}_{\mathrm{MT}_{i}}^{(k)}, \mathcal{A}_{\mathrm{MT}_{i}}\right)\right. \\
& \int_{\rho=0}^{\infty} \mathbb{E}_{R_{\mathrm{MT}_{i}}\left[\mathbf{1}\left(\mathcal{O}_{\mathrm{MT}_{i}}^{(j, k)}\right) \mathbf{1}\left(\mathcal{Z}_{\mathrm{MT}_{i}}\right)\right.} \\
& \left.\left.\frac{s(\tau \rho)^{-\alpha}\left(\tau R_{\mathrm{MT}_{i}}\right)^{\alpha \epsilon} p_{0}}{1+s(\tau \rho)^{-\alpha}\left(\tau R_{\mathrm{MT}_{i}}\right)^{\alpha \epsilon} p_{0}} \rho \mid \mathcal{X}_{\mathrm{MT}_{i}}^{(k, n)}, \mathcal{A}_{\mathrm{MT}_{i}}\right] \mathrm{~d} \rho\right)
\end{aligned}
$$

The next step is the computation of the expectation with respect to $R_{\mathrm{MT}_{i}}$ by conditioning on $\mathcal{X}_{\mathrm{MT}_{i}}^{(k, n)} \cap \mathcal{A}_{\mathrm{MT}_{i}}$ and by applying the definition of the event $\mathcal{O}_{\mathrm{MT}_{i}}^{(j, k)}$ in (5) and of $\mathcal{Z}_{\mathrm{MT}_{i}}$ in (6). In particular, by conditioning on $\mathcal{X}_{\mathrm{MT}_{i}}^{(k, n)} \cap \mathcal{A}_{\mathrm{MT}_{i}}$ for $\mathrm{MT}_{i} \in \Psi^{(k)}$, the distances $R_{\mathrm{MT}_{i}}$ are independent and identically distributed random variables whose PDF is in (11). With some algebra, $\mathcal{L}_{I}\left(s \mid \mathcal{X}_{\mathrm{MT}_{0}}^{(j)}\right)$ can be written as follows:

$$
\begin{aligned}
& \exp \left(-\sum_{k \in \mathcal{K}} 2 \pi \lambda^{(k)} \sum_{n \in \mathcal{K}} \operatorname{Pr}\left(\mathcal{Q}_{\mathrm{MT}_{i}}^{(n)} \mid \mathcal{X}_{\mathrm{MT}_{i}}^{(k)}, \mathcal{A}_{\mathrm{MT}_{i}}\right)\right. \\
& \int_{r=0}^{\infty} f_{R_{\mathrm{MT}_{i}}}\left(r \mid \mathcal{X}_{\mathrm{MT}_{i}}^{(k, n)}, \mathcal{A}_{\mathrm{MT}_{i}}\right) \int_{\rho=\max \left(\left(\frac{t^{(j)}}{t^{(k)}}\right)^{\frac{1}{\alpha}} r,\left(\frac{p_{0}}{i_{0}}\right)^{\frac{1}{\alpha}} \frac{(\tau r)^{\epsilon}}{\tau}\right)}^{\infty} \\
& \left.\frac{s(\tau \rho)^{-\alpha}(\tau r)^{\alpha \epsilon} p_{0}}{1+s(\tau \rho)^{-\alpha}(\tau r)^{\alpha \epsilon} p_{0}} \rho \mathrm{d} \rho \mathrm{d} r\right)
\end{aligned}
$$

The proof follows by computing the inner integral.

\section{Proof of Proposition 7}

The probability that a MT is muted is by definition as follows:

$$
\operatorname{Pr}\left(\mathcal{X}_{M T_{0}}^{(j, m)}, \overline{\mathcal{A}_{M T_{0}}}\right)=\operatorname{Pr}\left(\mathcal{X}_{M T_{0}}^{(j, m)}\right)-\operatorname{Pr}\left(\mathcal{X}_{M T_{0}}^{(j, m)}, \mathcal{A}_{M T_{0}}\right)
$$

where $\operatorname{Pr}\left(\mathcal{X}_{\mathrm{MT}_{0}}^{(j, m)}, \mathcal{A}_{\mathrm{MT}_{0}}\right)$ is known and $\operatorname{Pr}\left(\mathcal{X}_{M T_{0}}^{(j, m)}\right)$ is the probability that the MT is associated to tier $j$ and that the 
most interfered BS belongs to tier $m$. If $j \neq m$, it can be written as follows:

$$
\begin{aligned}
\operatorname{Pr} & \left(\mathcal{X}_{\mathrm{MT}_{0}}^{(j, m)}\right)=\mathbb{E}_{R_{\mathrm{MT}_{0},(1)}^{(m)}} \mathbb{E}_{R_{\mathrm{MT}_{0},(1)}^{(j)}, R_{\mathrm{MT}_{0},(2)}^{(j)}} \\
& {\left[\mathbf{1}\left(R_{\mathrm{MT}_{0},(1)}^{(m)}>\left(\frac{t^{(m)}}{t^{(j)}}\right)^{\frac{1}{\alpha}} R_{\mathrm{MT}_{0},(1)}^{(j)}\right) \times\right.} \\
& \left.\mathbf{1}\left(R_{\mathrm{MT}_{0},(1)}^{(m)}<R_{\mathrm{MT}_{0},(2)}^{(j)}\right)\right]
\end{aligned}
$$

The computation of this expectation is very similar to Appendix A, the PDF of the distance of the nearest BS and of the joint PDF of the distances of the nearest and second nearest BSs are needed. By definition of PPP, they are equal to $f_{R_{\mathrm{MT}_{0},(1)}^{(j)}}(r)=2 \pi \lambda^{(j)} r \mathrm{e}^{-\pi \lambda^{(j)} r^{2}}$ and

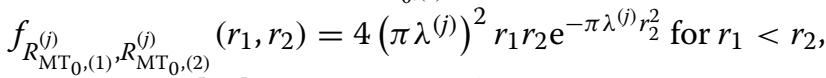
respectively, [21]. With the aid of these PDFs, we obtain:

$$
\begin{aligned}
& \operatorname{Pr}\left(\mathcal{X}_{M T_{0}}^{(j, m)}\right)=\int_{\max \left\{\left(\frac{t^{(m)}}{t^{(j)}}\right)^{\frac{1}{\alpha}} r_{1}^{(j)}, r_{1}^{(j)}\right\}}^{\infty} 2 \pi \lambda^{(m)} r_{1}^{(m)} \\
& \times \exp \left(-\pi \lambda^{(m)} r_{1}^{2(m)}\right) \int_{0}^{\infty} 2 \pi \lambda^{(j)} r_{1}^{(j)} \\
& \times\left(\exp \left(-\pi \lambda^{(j)} r_{1}^{2(j)}\right)-\exp \left(-\pi \lambda^{(j)} r_{1}^{2(m)}\right)\right) d r_{1}^{(m)} d r_{1}^{(j)}
\end{aligned}
$$

The computation of the two-fold integral leads to the function $\omega^{(j)}(v)$ that is provided in (44).

The case $j=m$ can be solved by using an approach similar to the previous case. The final result corresponds to the function $\zeta^{(j)}(v)$ available in (45).

By combining both cases $j=m$ and $j \neq m, \operatorname{Pr}\left(\mathcal{X}_{M T_{0}}^{(j, m)}\right)$ can be written as follows:

$$
\begin{aligned}
& \operatorname{Pr}\left(\mathcal{X}_{\mathrm{MT}_{0}}^{(j, m)}\right) \\
& =\int_{0}^{\infty}\left(\mathbf{1}(j \neq m) v^{(j, m)}(v)+\mathbf{1}(j=m) \eta^{(j)}(v)\right) \mathrm{d} v
\end{aligned}
$$

The proof follows by computing the summation over $m \in \mathcal{K}$ in (66).

\section{Proof of Lemma 6}

The Laplace transform of the interference for all the MTs can be expressed as follows:

$$
\begin{aligned}
& \mathcal{L}_{I_{\text {all }}}\left(s \mid \mathcal{X}_{\mathrm{MT}_{0}}^{(j)}\right)=\mathbb{E}_{I_{\text {all }}}\left[\mathrm{e}^{-s I_{\text {all }} \mid} \mathcal{X}_{\mathrm{MT}_{0}}^{(j)}\right]=\prod_{k \in \mathcal{K}} \mathbb{E}_{\Psi^{(k)}} \\
& \prod_{\mathrm{MT}_{i} \in \Psi^{(k)}} \mathbb{E}_{R_{\mathrm{MT}_{i}}}\left[\mathbb { E } _ { H _ { \mathrm { MT } _ { i } } } \operatorname { e x p } \left(-s H_{\mathrm{MT}_{i}}\left(\tau D_{\mathrm{MT}_{i}}\right)^{-\alpha}\right.\right. \\
& \left.\left.\left(\tau R_{\mathrm{MT}_{i}}\right)^{\alpha \epsilon} p_{0}\right) \mid \mathcal{X}_{\mathrm{MT}_{i}}^{(k)}\right]
\end{aligned}
$$

By applying the PGF theorem in [11] and computing the expectation with respect to the channel fading, $\mathcal{L}_{I_{\text {all }}}\left(s \mid \mathcal{X}_{\mathrm{MT}_{0}}^{(j)}\right)$ is as follows:

$$
\begin{aligned}
& \exp \left(-\sum_{k \in \mathcal{K}} 2 \pi \lambda^{(k)} \int_{\rho=0}^{\infty} \mathbb{E}_{R_{\mathrm{MT}_{i}}}\right. \\
& \left.\left[\frac{s(\tau \rho)^{-\alpha}\left(\tau R_{\mathrm{MT}_{i}}\right)^{\alpha \epsilon} p_{0}}{1+s(\tau \rho)^{-\alpha}\left(\tau R_{\mathrm{MT}_{i}}\right)^{\alpha \epsilon} p_{0}} \rho \mid \mathcal{X}_{\mathrm{MT}_{i}}^{(k)}\right] \mathrm{d} \rho\right)
\end{aligned}
$$

By conditioning on the event $\mathcal{Q}_{\mathrm{MT}_{i}}^{(n)}$ defined in (2) and by using the total probability theorem, $\mathcal{L}_{I}\left(s \mid \mathcal{X}_{\mathrm{MT}_{0}}^{(j)}\right)$ can be written as follows:

$$
\begin{aligned}
& \exp \left(-\sum_{k \in \mathcal{K}} 2 \pi \lambda^{(k)} \sum_{n \in \mathcal{K}} \operatorname{Pr}\left(\mathcal{Q}_{\mathrm{MT}_{i}}^{(n)} \mid \mathcal{X}_{\mathrm{MT}_{i}}^{(k)}\right) \int_{\rho=0}^{\infty} \mathbb{E}_{R_{\mathrm{MT}_{i}}}\right. \\
& \left.\left[\frac{s(\tau \rho)^{-\alpha}\left(\tau R_{\mathrm{MT}_{i}}\right)^{\alpha \epsilon} p_{0}}{1+s(\tau \rho)^{-\alpha}\left(\tau R_{\mathrm{MT}_{i}}\right)^{\alpha \epsilon} p_{0}} \rho \mid \mathcal{X}_{\mathrm{MT}_{i}}^{(k, n)}\right] \mathrm{d} \rho\right)
\end{aligned}
$$

The next step is the computation of the expectation with respect to $R_{\mathrm{MT}_{i}}$ by conditioning on $\mathcal{X}_{\mathrm{MT}_{i}}^{(k, n)}$. In particular, by conditioning on $\mathcal{X}_{\mathrm{MT}_{i}}^{(k, n)}$ for $\mathrm{MT}_{i} \in \Psi^{(k)}$, the distances $R_{\mathrm{MT}_{i}}$ are independent and identically distributed random variables whose PDF is in (46). With some algebra, $\mathcal{L}_{I_{\text {all }}}\left(s \mid \mathcal{X}_{\mathrm{MT}_{0}}^{(j)}\right)$ can be written as follows:

$$
\begin{aligned}
& \exp \left(-\sum_{k \in \mathcal{K}} 2 \pi \lambda^{(k)} \sum_{n \in \mathcal{K}} \operatorname{Pr}\left(\mathcal{Q}_{\mathrm{MT}_{i}}^{(n)} \mid \mathcal{X}_{\mathrm{MT}_{i}}^{(k)}\right)\right. \\
& \int_{r=0}^{\infty} f_{R_{\mathrm{MT}_{i}}}\left(r \mid \mathcal{X}_{\mathrm{MT}_{i}}^{(k, n)}\right) \int_{\rho=r}^{\infty} \\
& \left.\frac{s(\tau \rho)^{-\alpha}(\tau r)^{\alpha \epsilon} p_{0}}{1+s(\tau \rho)^{-\alpha}(\tau r)^{\alpha \epsilon} p_{0}} \rho \mathrm{d} \rho \mathrm{d} r\right)
\end{aligned}
$$

The proof follows by computing the inner integral. 
Acknowledgements

Not applicable.

\section{Funding}

This work was supported in part by the European Commission through the H2020-MSCA ETN-5Gaura project under Grant Agreement 675806.

\section{Availability of data and materials}

Data sharing is not applicable to this article as no datasets were generated or analysed during the current study. The paper is built upon mathematical analysis.

\section{Authors' contributions}

The authors declare that they have equally contributed to the paper. All authors read and approved the final manuscript.

\section{Competing interests}

The authors declare that they have no competing interests.

\section{Publisher's Note}

Springer Nature remains neutral with regard to jurisdictional claims in published maps and institutional affiliations.

\section{Author details}

1 'Departamento de Ingeniería de Comunicaciones, Universidad de Málaga, Málaga, Avda. Cervantes, 2, 29071 Málaga, Spain. ² Laboratoire des Signaux et Systèmes, CNRS, CentraleSupelec, Univ Paris-Sud, Université Paris-Saclay, Paris, Gif-sur-Yvette, 91192 Paris, France. ${ }^{3}$ Keysight Technologies, Calle Severo Ochoa, 21, 29590 Málaga, Spain.

Received: 21 December 2018 Accepted: 13 March 2019

Published online: 25 April 2019

\section{References}

1. H. Elshaer, F. Boccardi, M. Dohler, R. Irmer, in 2014 IEEE Global Communications Conference. Downlink and uplink decoupling: a disruptive architectural design for 5 G networks, (2014), pp. 1798-1803. https://doi.org/10.1109/GLOCOM.2014.7037069

2. S. Singh, X. Zhang, J. G. Andrews, Joint rate and SINR coverage analysis for decoupled uplink-downlink biased cell associations in HetNets. IEEE Trans. Wirel. Commun. 14(10), 5360-5373 (2015). https://doi.org/10.1109/ TWC.2015.2437378

3. T. D. Novlan, H. S. Dhillon, J. G. Andrews, Analytical modeling of uplink cellular networks. IEEE Trans. Wirel. Commun. 12(6), 2669-2679 (2013). https://doi.org/10.1109/TWC.2013.050613.120325

4. H. ElSawy, E. Hossain, On stochastic geometry modeling of cellular uplink transmission with truncated channel inversion power control. IEEE Trans. Wirel. Commun. 13(8), 4454-4469 (2014). https://doi.org/10.1109/TWC. 2014.2316519

5. M. D. Renzo, P. Guan, Stochastic geometry modeling and system-level analysis of uplink heterogeneous cellular networks with multi-antenna base stations. IEEE Trans. Commun. 64(6), 2453-2476 (2016). https://doi. org/10.1109/TCOMM.2016.2552163

6. F. J. Martin-Vega, et al., Analytical modeling of interference aware power control for the uplink of heterogeneous cellular networks. IEEE Trans. Wirel. Commun. PP(99), 1-1 (2016). https://doi.org/10.1109/TWC.2016. 2588469

7. H. Zhang, N. Prasad, S. Rangarajan, S. Mekhail, S. Said, R. Arnott, in Communications (ICC), 2012 IEEE International Conference On. Standards-compliant LTE and LTE-A uplink power control, (2012), pp. 5275-5279. https://doi.org/10.1109/ICC.2012.6364333

8. H. Shin, J. H. Lee, Channel reliability estimation for turbo decoding in rayleigh fading channels with imperfect channel estimates. IEEE Commun. Lett. 6(11), 503-505 (2002). https://doi.org/10.1109/LCOMM.2002.804246

9. M. C. Aguayo-Torres, et al., WM-SIM LTE link simulator. Technical report. (University of Malaga, 2014). http://riuma.uma.es/xmlui/handle/10630/ 7438

10. F. J. Martin-Vega, I. M. Delgado-Luque, F. Blanquez-Casado, G. Gomez, M. C. Aguayo-Torres, J.T. Entrambasaguas, in Vehicular Technology Conference (VTC Fall), IEEE 78th. LTE performance over high speed railway channel, (2013). https://doi.org/10.1109/NTCFall.2013.6692274

11. M. Haenggi, Stochastic geometry for wireless networks. (Cambridge University Press, 2013)
12. H. S. Dhillon, J. G. Andrews, Downlink rate distribution in heterogeneous cellular networks under generalized cell selection. Wirel. Commun. Lett. IEEE. 3(1), 42-45 (2014). https://doi.org/10.1109/WCL.2013.110713.130709

13. J. G. Andrews, F. Baccelli, R. K. Ganti, A tractable approach to coverage and rate in cellular networks. IEEE Trans. Commun. 59(11), 3122-3134 (2011). https://doi.org/10.1109/TCOMM.2011.100411.100541

14. S. Sesia, et al., LTE, The UMTS long term evolution: from theory to practice. (Wiley Publishing, 2009)

15. M. D. Renzo, et al., The intensity matching approach: a tractable stochastic geometry approximation to system-level analysis of cellular networks. IEEE Trans. Wirel. Commun. 15(9) (2016). https://doi.org/10.1109/TWC 2016.2574852

16. S. Singh, F. Baccelli, J. G. Andrews, On association cells in random heterogeneous networks. Wirel. Commun. Lett. IEEE. 3(1), 70-73 (2014). https://doi.org/10.1109/WCL.2013.111713.130707

17. J.-S. Ferenc, Z. Néda, On the size distribution of Poisson Voronoi cells. Physica A: Statistical Mechanics and its Applications. 385(2), 518-526 (2007)

18. 3GPP, Technical specification group radio access network; Small-cell enhancements for E-UTRA and E-UTRAN - Physical layer aspects. TR 36.872. 3rd Generation Partnership Project (3GPP) (2013)

19. S. Singh, J. G. Andrews, Joint resource partitioning and offloading in heterogeneous cellular networks. IEEE Trans. Wirel. Commun. 13(2), 888-901 (2014). https://doi.org/10.1109/TWC.2013.120713.130548

20. Y. Lin, W. Bao, W. Yu, B. Liang, Optimizing user association and spectrum allocation in HetNets: a utility perspective. IEEE J. Sel. Areas Commun. 33(6), 1025-1039 (2015). https://doi.org/10.1109/JSAC.2015.2417011

21. F. J. Martin-Vega, F. J. Lopez-Martinez, G. Gomez, M. C. Aguayo-Torres, in Global Communications Conference (GLOBECOM), 2014. Multi-user coverage probability of uplink cellular systems: A stochastic geometry approach (IEEE, 2014). https://doi.org/10.1109/GLOCOM.2014.7037431

\section{Submit your manuscript to a SpringerOpen ${ }^{\circ}$ journal and benefit from:}

- Convenient online submission

Rigorous peer review

- Open access: articles freely available online

- High visibility within the field

- Retaining the copyright to your article

Submit your next manuscript at $\gg$ springeropen.com 